\title{
An equity-oriented multi-objective inventory management model for blood banks considering the patient condition: A real-life case
}

\author{
Mahnaz Sohrabi a ${ }^{\text {a }}$ Ph.D. Candidate \\ ${ }^{a}$ Faculty of Industrial and Mechanical Engineering, Qazvin Branch, Islamic Azad University, \\ Qazvin, Iran. Tel: +989128083610 \\ E-mail: m.sohrabi@qiau.ac.ir (https://orcid.org/0000-0002-3037-6418)
}

Mostafa Zandieh ${ }^{* b}$, Ph.D.

b Department of Industrial Management and Information Technology, Management and Accounting Faculty, Shahid Beheshti University, Tehran, Iran. Tel: +9829905215

E-mail: $\underline{\text { m_zandieh@sbu.ac.ir (https://orcid.org/0000-0003-1209-9514) }}$

Behrouz Afshar-Nadjafi ${ }^{a}$, Ph.D.

${ }^{a}$ Faculty of Industrial and Mechanical Engineering, Qazvin Branch, Islamic Azad University, Qazvin, Iran. Tel: +989125817105

E-mail: afsharnb@alum.sharif.edu (https://orcid.org/0000-0002-3391-8411) 


\title{
An equity-oriented multi-objective inventory management model for blood banks considering the patient condition: A real-life case
}

\begin{abstract}
The absence of systematic disparities in health utilization leads to achieving equity in health. However, equity in delivering healthcare services is always challenging because of financial and medical resource constraints. In this regard, a practical multi-objective mixed-integer linear programming model with priority-differentiated demand classes is presented for cost-effective inventory management of blood products considering health equity. The system deals with multiple substitutable products. There are elective and non-elective demands, which are categorized into three main classes based on medical urgencies. The health objectives are investigated to achieve a desirable health equity level in delivering healthcare services to patients. Moreover, the economic objective is pursued to minimize total costs incurred across managing the inventory without weakening the service level. An effective demand-oriented hybrid heuristic is proposed to issue and allocate the blood for demand satisfaction equitably. A goal programming approach is utilized to find the optimum solution. The applicability of the model is validated through a real case study. Finally, several sensitivity analyses are conducted to gain useful managerial insights. According to the results, the proposed model presents a proper solution by making a reasonable health-economic trade-off. Also, the results illustrate the beneficial improvement in patient care and promoting health equity.
\end{abstract}

Keywords: Blood banking; Blood supply chain; Emergency demands; Health equity; Inventory management; Medical urgent levels; Perishable items; Priority-differentiated demands.

\section{Introduction}

Health equity is an essential criterion of health services. Equity in health implies that healthcare systems should ideally provide a fair opportunity for everyone to attain their full health potential [1]. In this regard, healthcare systems mainly focus on delivering the best quality of service for patients. However, increasing population age, recent medical advances, more aggressive treatment of some diseases, and the growing need for various medicines have made it challenging to provide the highest medical services and products required by the health system [2]. This issue can be very challenging because there are different ways to define equity, evaluate its effects, and grouping patients to analyze health equity. Therefore, a definition is needed to be operationalized based on measurable criteria [3]. For healthcare systems, social equity entails that a patient is appropriately treated in various aspects of the disease. This requirement is consistent with a familiar distinction between horizontal and vertical social equity [4]. In healthcare systems, horizontal social equity requires similar medical services for similar patients. On the other hand, vertical social equity entails dissimilar medical services for dissimilar patients in the aspect of urgent levels (ULs) and commensurate with their illness [5].

Healthcare supply chain management may be considered more complex than typical industrial applications such as the food supply chain. In healthcare, each product item may be considered crucial. Besides, there is a perceived need to supply very high service levels. On the other hand, there is a high production value and, in many cases, a need for special handling to minimize 
expiration or spoilage. Thus, logistics and inventory management costs can be substantial [6]. In the meantime, controlling blood products' inventory, especially blood platelet, is much more difficult [7]. The main challenge is a rare and limited blood supply, inadequate access to donors, very limited shelf-lives, and regular use in treating certain diseases such as cancer [8]. These issues should be eliminated to provide a desirable health service to patients. Simultaneously, the least possible shortage and wastage happen [9].

The healthcare administrators aim to provide demand-oriented services and making them accessible for various patients groups. Moreover, they aim to minimize the total system costs by adopting cost-efficient policies, followed by shortage reduction without any wastage or failure [10]. In this regard, the healthcare managers need to choose efficacious policies to serve the patients properly and keep the blood inventories at an optimum level. Implementing appropriate policies for the equitable assignment of health services in a way that minimizes health risks and operating costs is one of the main concerns of decision-makers [11,12].

Although meeting demand is of paramount importance, the operational cost of handling blood products cannot be ignored [8]. Due to the rapid growth in healthcare expenditure worldwide and simultaneous growth of demand for healthcare services, developing efficient and effective healthcare systems has become an essential concern for governments and healthcare managers [13]. An increase in safety measures, new donor recruitment programs, a surge in demand for blood products, and inefficient usage of resources was cited as the possible reasons for this cost increase [14]. Thus, society must improve utilization within blood supply chains and reduce costs to make the best use of limited blood resources [15].

Blood comprises several components such as red blood cells (RBC), platelets, and plasma with different shelf-life and applications. Depending on the patient's condition, whole blood or one of its components may be needed for treatment [16]. Moreover, there are a diversity of blood types in eight blood groups, namely $\mathrm{ABO}$ and $\mathrm{Rh}[17,18]$. All blood types of a particular blood component have the same shelf-life. Usually, the patient's blood requirement is met with the same blood type. Note that blood substitution is only allowed among different blood types (not different components) based on the medical priorities and the physician's decision in case of shortage [16].

The focus point of successful blood supply chain management is inventory control [19,20]. Inventory management is a part of more extensive operations. It plays a supportive and active role in improving the overall logistics and supply chain performance. The utilization of streamlined inventory management techniques can significantly impact making the blood baking operations more efficient to a large extent. Even though inventory management systems use cost-based models, hospitals need to focus on the patient service level. Availability of healthcare items of high quality in a continuously fluctuating environment is challenging [15].

In this regard, a systematic inventory management approach and timely use of medical products before their expiration date can dramatically help the healthcare system to spend its financial resources more economically through removing redundant costs without weakening the level of offered healthcare services [20]. The current study aims to find a proper solution for the decisionmakers by proposing a health-economic inventory management model. 
In this paper, a replenishment policy for inventory management of blood banks is studied. Also, an issuance and allocation rule based on the current practices on substitutable and perishable products is proposed. In doing so, we can develop an effective heuristic policy and generate insights for the general problem. Moreover, the current study discusses the health-economic challenges of blood banks. In this respect, two main objectives are pursued: first, health equity in delivering blood products to different patient groups; second, the optimal management of blood products to minimize the risks of lives and economic costs.

This paper seeks to answer the following questions: What strategies to be adopted for minimizing the outdated blood units without facing any shortages? How much of a specific blood product and in which planning periods should be replenished? Which demand should be met first to observe the health equity and the desired level of medical services on the one hand, and the least amount of shortage occurs on the other hand? How does the system deal with the real-world environment challenges and emergency demands to pose the least risk to the patients' lives? How to issue an inventory from the blood bank and allocate it to demand so that the least amount of wastage occurs?

The rest of this paper is organized as follows: Section 2 reviews the related literature. The applied methodology and the proposed mathematical model are described in Sections 3 and 4, respectively. Section 5 investigates a real case study, including solutions, obtained results, and discussion over the findings. Moreover, some sensitivity analyses are conducted over some of the critical parameters in this section. Finally, Section 6 presents the conclusion and future streams.

\section{Literature review}

A review of the most related papers in blood inventory management and health equity literature is conducted in this section. Finally, the research gap and the main contributions of the current study are mentioned. A summary of the recently published papers on the related topics is provided in Table 1 and discussed in the following.

\subsection{Related works}

In this section, the most related papers are studied in terms of problem domains, modeling, and solution methods. There is an extensive body of research in the blood supply chain (BSC). Most research in this field has focused mainly on minimizing inventory costs, shortage, and wastage using various modeling tools and techniques to increase efficiency and effectiveness in the overall management of the BSC [20,21]. In this regard, replenishment policies are used to determine the optimal ordering quantity and replenishment periods. In this regard, various studies considered classical or modified periodic review [9,22,23] and continuous review [24,25] policies. Considering the lead-time as zero or constant is a logical and common approximation for modeling such healthcare systems [26-28].

In addition to the replenishment policy, the issuance policy also affects the inventory shortage and wastage [29]. The two policies of first-in-first-out (FIFO) for the consumption of older products 
[26,30] and last-in-first-out (LIFO) for the consumption of fresher [30] had the highest application in the literature. Besides, some studies have suggested modified [29], hybrid and heuristic policies [9]. Ideally, it is better to consume older products earlier to avoid their wastage, but this is not always possible. For blood products, the issuance policy may change depending on medical preferences and patient conditions. This matter results in sorting out the demands for a given product according to their required age (or remained shelf-life) and the order of meeting [26].

As Table 1 addresses, single or multiple objectives are considered in the literature. The modeling methods can be categorized into mathematical programming, simulation, and other methods. The mathematical programming methods mainly consist of mixed-integer linear programming (MILP) models and mixed integer nonlinear programming (MINLP) models. Studies that developed mathematical modeling, in most cases, use exacts, heuristics, and metaheuristic solution approaches [31]. Most extended mathematical models in blood inventory management have been conducted in a deterministic environment because of the complexity of coping with uncertainty [32]. However, more attention has been paid to BSC uncertainties in the last two decades. In general, three types of uncertain environments of stochastic, fuzzy, and robust are defined. In this regard, different or hybrid approaches are used to model them [2].

The importance of blood freshness for some treatments has been reported in many studies $[8,16,26]$. For example, platelet demand from organ transplant patients is treated as an emergency, and receiving the youngest possible blood platelets. Oncology patients request the youngest platelets, and traumatology patients have no preference concerning the blood platelet's age [33]. Additionally, the eldest red blood cells' transfusion impacts short- and long-term survival rates for cardiac patients [8].

In summary, certain patients require the freshest available platelets to minimize complications during treatment. In contrast, the risk of complication for other patients is believed to be minimal. This paper proposes a model with an age-differentiated demand to reflect findings in the literature and the current practice in blood management. In this case, classical FIFO and LIFO policies may not necessarily be appropriate to meet the patients' needs. Therefore, it is better to consider agebased issuance policies to manage perishable inventories. In this regard, first-expired-first-out (FEFO) and last-expired-first-out (LEFO) policies are suggested [8,34].

Inventory control systems are recommended to be aligned with the patient's condition. Technically and scientifically, healthcare items' demand is closely linked to physician recommendations based on patient conditions [13]. In healthcare systems, patient conditions are divided into three categories based on urgency: immediate/severe, moderate/delayed, and minor [10]. In BSC, a specific product can be prescribed to treat diseases in different urgent classes $[8,10]$. The demand is classified based on the requested blood product's age in some previous works $[8,16,26]$. However, according to the authors' knowledge, there are no relevant studies on demand classification based on medical urgencies in the field of blood inventory management.

Based on the report of a recent study on a real blood bank process, the blood demands are generally divided into two main categories in the aspect of election administration: elective (scheduled) and non-elective (non-scheduled) [35]. However, the hospitals mostly encounter non-elective demands 
in case of emergencies. The non-elective demands mainly arise in emergency rooms, intensive care units, and operating rooms [13]. Hospitals' current practice is to delay elective surgeries when the inventory on hand is limited [8]. In the literature, the emergency condition has often been overlooked. Some of the previous studies considered the emergency as an instance of a shortage in the system. In this case, the required inventory is met through an emergency or expedited order $[20,31]$. The emergency demands are typically lower than scheduled ones. Note that the emergency demand might occur among elective and/or non-elective demands. This kind of shortage can hurt the blood bank's reputation, so more optimization must be done to prepare for emergency conditions [9].

Regarding the diversity of blood types (ABO), physicians prefer to use the same blood type for patients in the first place. Nevertheless, in cases of shortage, substitution is a possibility based on medical priorities [35]. Most previous models are generally limited to one or two non-substitutable products for more simplification $[8,20,26]$. However, in recent years, the substitution possibility among blood types has received more attention [16].

Several papers are focused on managing blood at multiple locations with possible transshipment options. In this case, a lateral transshipment policy is considered [28]. In practice, there is no trading of blood platelets between different blood banks (due to the platelets' high perishability). However, hospitals can get fresher platelets from other blood centers or hospitals in case of shortage, especially for emergencies. In this case, an expedited replenishment takes place [33]. The same assumption is made in this paper to get closer to real-life conditions. Note that this assumption technically has no difference from assuming "lost demand" in inventory management. The excess demand does not impact either the inventory composition or the usage in future periods. Although, a penalty cost is incurred for each unit of demand that cannot be satisfied immediately from inventory on hand [8].

Some researchers have described equity in health as an essential criterion of services and the level of access to public health services [10]. Using a supply-demand framework, they discussed key challenges in achieving a health system that provides equity in service delivery, health financing, and financial risk protection. Heretofore, many healthcare researchers have conceptually described social equity for medical services $[1,4,5]$. Because of health equity's hierarchical complexity, few healthcare researchers have considered health equity and patients' medical priorities in their quantitative studies so far [36].

The review of the existing literature shows that most of the previous studies in this area were qualitative. To the best of the authors' knowledge, few papers have pragmatically focused on social equity implementation by operational research approaches to optimize healthcare performance. In recent work, the BSC's social equity was considered for three types of injuries, differentiated in terms of medical ULs and demand satisfaction preferences [10]. In another research in this field, to make an equitable health service network redesign, equity is calculated by the most urgent need of a patient from a medical viewpoint and transportation time from the logistics viewpoint [2]. To the best of the authors' knowledge, the blood inventory management model considering health equity has not been formulated so far. 


\subsection{Research gap and contributions}

Blood inventory optimization is a critical point for blood services because expenditures must be strictly controlled while maintaining high medical service levels. This paper is motivated to examine blood inventories health-economic optimization when a hospital blood bank is responsible for blood purchases and logistics to internal wards.

This study adds further credibility to the importance of applying a practical approach for managing blood inventories optimally by developing an equity-oriented demand-oriented mathematical model for healthcare systems. The elective and non-elective demands are considered and classified into three classes in aspects of emergencies. The product freshness, substitution allowance, response urgency, and allocation policy are specified according to each patient group's condition and blood bank's current practice. Note that the authors have investigated the understudy problem and assumptions practically in a previous study related to the real hospitals' blood banks process model [35].

To the best of the authors' knowledge, this paper is the first pragmatic study to address health equity in blood inventory management as one of the main criteria in satisfying the increasing demands. Altogether, this paper is conducted to bridge the literature's gap and can be differentiated from the previous blood inventory management studies by bringing together the following main contributions:

- Implementing health equity in blood inventory management for the first time to provide a desirable level of accessibility of medical services for all patients' groups based on medical priorities;

- Making a trade-off between the equity and economic objectives of healthcare systems;

- Considering elective and non-elective demands and divided them into three priority classes based on blood age, substitution allowance, medical urgency, and allocation policy;

- Adopting a practical policy to meet the emergency demands;

- Implementing a hybrid heuristic policy to issue and assign the blood inventory so that the least shortage and wastage occurs;

- Addressing a practical perspective for the planning of a multi-product inventory management system with substitution allowance, limited supply resources, and storage capacity constraint over a multi-period horizon;

- Applying a real-life case to demonstrate the wide practicality of the proposed problem in healthcare systems.

\section{Methodology}

The proposed model in this research is a multi-objective mixed-integer linear programming (MOMILP) model. The objectives should be satisfied efficiently in a non-dominance space by finding global Pareto-optimal solutions. In the first step, a mathematical model is proposed. Then an extended goal programming (GP) approach is employed to solve it in an actual situation. Next, the GP approach is described for making a trade-off among different objectives of the proposed model.

Different approaches have been proposed in the literature to solve the bi- or multi-objective decision-making (MODM) optimization problems. The methods applied for combining the 
objective functions into only one. GP is an old yet applicable solution method among available ones. This approach is used when the number of objectives is too much, while their importance is mostly the same [37]. It is selected to solve our multi-objective inventory management problem due to its close relation with linear programming [38]. Minimizing shortage as well as inventory level, substitution, non-freshness, non-response to non-elective demand, and total system costs are goals of this problem. Naturally, some goals may contradict others which their associate weights determine their importance. When decision-makers can easily determine each goal's weight, the GP approach seems appropriate [38]. That is why the GP approach is selected.

A goal is first defined for every objective, ranging between 0 and 1, to implement the GP approach. The objective function tries to minimize the maximum deviation from each goal. Generally, a typical MODM problem is defined as follows [39]:

$$
\left\{\begin{array}{c}
\operatorname{Min}\left(\mathrm{f}_{1}(\mathrm{x}), \mathrm{f}_{2}(\mathrm{x}), \ldots, \mathrm{f}_{\mathrm{n}}(\mathrm{x})\right) \\
x \in X
\end{array}\right.
$$

Given that the optimal value of each objective is shown by $f_{i}{ }^{*}$, where $i=1,2, \ldots, n$. In real-world MODM problems, taking existing conflict into account among objectives, there is no solution $x^{*} \in X$, for which all objectives are optimized. Accordingly, if $A$ is a solution method and $x^{A}$ is its output, then this solution approach is more efficient when $f_{i}\left(x^{A}\right)$ has less distance to $f_{i}{ }^{*}$.

In the GP approach, an acceptable value is first considered for every objective (e.g., its optimal value $\left.f_{i}^{*}\right)$. Then, the goal function is defined so that by any reduction in the objective function, the goal value is linearly increased. Supposed that $m_{i}=f_{i}^{*}$ is considered as the minimum/ideal value of $i^{\text {th }}$ objective and $M_{i}$ is defined as its upper bound. So, in the GP method, the $i^{\text {th }}$ objective could be defined as follows [38]:

$$
G_{i}(x)=G\left(f_{i}(x)\right)=\left\{\begin{array}{l}
0 ; f_{i}(x) \geq M_{i} \\
\frac{M_{i}-f_{i}(x)}{M_{i}-m_{i}} \\
1 ; f_{i}(x) \leq m_{i}
\end{array} ; x \in X\right.
$$

Then, using the GP method, MODM problems could be stated in terms of a single objective model, as follows:

$$
\left\{\begin{array}{c}
\max z=\min \left\{G_{i}(x): i=1: n\right\} \\
G_{i}(x)=\frac{M_{i}-f_{i}(x)}{M_{i}-m_{i}} ; i=1,2, \ldots, n \\
x \in X
\end{array}\right.
$$

Where, if $f_{i}(x) \rightarrow m_{i}$, then, $G_{i}(x) \rightarrow 1$. 
In some cases, instead of the formulation mentioned above, the minimax technique is used. This technique is employed in this research as well. In the minimax method, the maximum deviation from the optimal value of the objectives is minimized. The formulation of the minimax method could be stated as follows [40]:

$$
\left\{\begin{array}{c}
G P \\
\min L_{\infty}\left(d_{i}(x), i=1,2, \ldots, n\right) \\
d_{i}(x)=\frac{f_{i}(x)-m_{i}}{M_{i}-m_{i}} ; i=1,2, \ldots, n \\
x \in X
\end{array}\right.
$$

Where, $L_{\infty}$ signifies the extreme norm and $d_{i}(x)=\frac{f_{i}(x)-m_{i}}{M_{i}-m_{i}}$ is the relative deviation of each objective function from its optimal value. The formulation mentioned above could be linearized as follows [40]:

$$
\left\{\begin{array}{c}
G P \\
\min Z \\
Z \geq \frac{f_{i}(x)-m_{i}}{M_{i}-m_{i}} ; i=1,2, \ldots, n \\
x \in X
\end{array}\right.
$$

\section{Problem statement and mathematical modeling}

This study is designed according to the current concerns of a typical blood bank [35]. The proposed system aims to provide efficient equity-oriented strategies for blood banks [12]. Accordingly, taking the importance of human lives into account, healthcare systems mainly deal with health equity concerns in service delivery so that the most negligible health risks happen. The current investigation seeks to find optimal ways to meet the blood demands health-economically, with minimal shortage and wastage. The problem statement is presented in the following.

\subsection{Problem statement}

In this research, the demands are divided into two general groups consist of elective demand and non-elective demand. Elective demands are scheduled based on previous physicians' requests. Non-elective demands are unscheduled demands. The demands are also separated into three priorities based on medical urgencies classes at the physician's discretion and considering social equity, named "High", "Moderate", and "Low" (i.e., high priority demand to low priority demand). The patients at each class have a specific medical condition and treatment urgency. Both elective and non-elective demands can fall into any of the above three classes. Figure 1 illustrates the demands' classes specifications graphically in summary. 
The product items in their first half-life are considered fresh. Those items which have passed their first half-life are considered ordinary (regular) in the aspect of freshness. At the end of a product item's shelf-life, it is expired and discarded. The demands are periodically (daily) and independent. Figure 2 indicates the blood inventory flow in the proposed system [35].

The demand classes are differentiated by UL, substitution allowance, freshness value, and allocation policy. Equity-oriented treatment prioritizes demand satisfaction not to be the same at the differentiated demands class. In this regard, a hybrid heuristic policy is presented for demand satisfaction. Suppose the demand for a given product cannot be satisfied with the same product type due to shortage. In that case, one can consider a shortage-based substitution policy, according to the patient's UL, or use expedited service for an immediate replenishment.

In the case of expedited replenishment, a penalty is imposed on the system. After employing the extended allocation and issuance hybrid policies, if some parts of demands have not yet been satisfied, they would be lost. The supply is capacitated and carried out only by one leading supplier. Also, the products received from the supplier have a specific shelf-life. The following is presented on how to meet the demand for different levels of urgency:

- Class 1: The highest priority demand is only for the freshest items due to avoiding medical risk. The same item could only meet the product demand at this level, so substitution in this UL is not allowed. It would be lost and ordered immediately by imposing an expedited service penalty cost in case of a shortage. An appropriate issuance policy, so-called the LEFO, is considered for this UL to assign the freshest items to the demand.

- Class 2: The moderate priority demand has lower medical sensitivity than class 1 , but there is still some medical risk; therefore, similar to class 1 , this level belongs to the fresh items but follows a different pattern. The issuance policy for satisfying demands in class 2 is a mix of the FEFO and LEFO policies. Firstly, the demand is met with fresh items in descending order. In this case, the FEFO issuance policy is used for demand satisfaction. According to the medical priorities, shortage-based substitution with the freshest items of other types of products is allowed at this level and follows the LEFO issuance policy. If there is still some unmet demand after the substitution, it would be lost and ordered immediately through imposing expedited service penalty costs.

- Class 3: The lowest priority demand encounters the least level of health risk and medical sensitivity. So, the products' remaining shelf-life is not that important for demand satisfaction; however, it is preferred to use the fresher ones to treat patients. Suppose some parts of the demands could not be met. In that case, shortage-based substitution with other types of products is allowed, according to the medical priorities. If some demand parts are still unsatisfied, the shortage would be lost. The optimal policy (OP) issuance policy is considered in this case. 


\subsubsection{Assumptions}

The following assumptions are considered for this investigation:

- The planning horizon is finite, including some specific periods (e.g., one-month plan with daily periods).

- The supplier capacity is limited and variable in each period.

- The demand for each product varies in each period.

- The shelf-life of all products is the same.

- The products are received in the freshest form from the supplier.

- The medical system's storage capacity is limited, and a maximum holding capacity is already determined for each product.

- The allowed ordering periods are already specified and are a subset of the planning horizon periods.

- The demand back-order is not allowed, and each period's demand must be satisfied in the same period, not the next.

- The products at their first half-life are considered fresh, and in their second half-life, they are considered ordinary.

- Medical experts determine the importance of product availability for each UL, ranging between 5 and 10 (the number 10 is the most important).

- The substitution rate of products ranges between 0 and 1 , specified by medical experts; 1 signifies fully compatible, and 0 means non-compatible.

- Each product's freshness importance in each UL ranges between 5 and 10, specified by medical experts (the number 10 is the most important).

- Lead-time to receive the order from the supplier is deterministic (one period). Also, expedited orders are received immediately in the same period from the supplier.

- The initial inventory of the system is negligible.

\subsubsection{Notations}

The notations and symbols used in the proposed mathematical model are as follows. Next, the mathematical formulation is presented.

\section{Sets and indices}

$T$ : Set of planning periods (day), $T=\{1,2, \ldots, t, \ldots, T \mid\}$

$R$ : Allowed replenishment periods (day), $R \subseteq T$

$P$ : Set of product types, $P=\{1,2, \ldots, p, \ldots,|P|\}$

$U:$ Set of demand priorities (demand classes), $U=\{1,2, \ldots, u, \ldots,|U|\}$

$S^{p p^{\prime}}$ : Set of substitutable products, product $p$ can be substitute with the product $p^{\prime}, S^{p p^{\prime}} \subseteq P$

$I$ : Set of products shelf-life (day), $I=\{1,2, \ldots, i, \ldots,|I|\}$ 


\section{Parameters}

$F C$ : Fixed-cost of purchasing products (currency/unit)

$P C_{p t}$ : The variable purchasing cost of product $p$ at period $t$ (currency/unit)

$S C_{p t}$ : Expedited service cost of product $p$ at period $t$ (currency/unit)

$H C_{p i}$ : Inventory holding cost of product $p$ with age $i$ (currency/unit)

$E C_{p}:$ Cost of expired/outdated products (currency/unit)

$E D_{p u t}$ : Elective-demand for product $p$ with priority $u$ at period $t$ (unit)

$N D_{p u t}$ : Non-elective demand for product $p$ with priority $u$ at period $t$ (unit)

$c_{p t}$ : Maximum supplier capacity to provide product $p$ at period $t$ (unit)

$v$ : Total storage capacity of the medical system (unit)

$m_{p}$ : The storage capacity of the medical center for one unit of product $p$ (unit)

$m v_{p}:$ The maximum storage capacity of the medical system for product $p$ (unit)

$\eta_{p u}$ : The relative importance of the availability of product $p$ for patients with priority $u$

$\pi_{p u}$ : The relative importance of freshness of product $p$ for patients with priority $u$

$\theta_{p p^{\prime} u}:$ Substitution rate of product $p$ with product $p$ ' for demand with priority $u$

$\delta_{p u}: 1$ if it is possible for demand with priority $u$ to substitute product $p$ with another product; otherwise, 0

\section{Decision variables and outputs}

$Q_{p t}$ : The order quantity for product $p$ at period $t$ (unit)

$I_{p i t}:$ Inventory level of product $p$ with age $i$ at period $t$ (unit)

$A_{p i t}$ : Number of assigned/consumed inventories of product $p$ with age $i$ for total demands satisfaction at period $t$ (unit)

$F_{\text {piut }}$ : Number of assigned/consumed inventories of product $p$ with age $i$ for satisfying demand with priority $u$ at period $t$ (unit)

$F_{p i p \text { ut }}^{\prime}$ : Amount of substitute inventories of product $p$ with age $i$ for satisfying demand of product $p^{\prime}$ with priority $u$ at period $t$ (unit)

$D_{p u t}$ : Total satisfied the demand for product $p$ with priority $u$ at period $t$ (unit)

$B_{p u t}$ : Total unfulfilled demand of product $p$ with priority $u$ at period $t$, before expedited orders (unit)

$X_{p u t}$ : Number of expedited orders for product $p$ with priority $u$ at period $t$ (unit)

$Z_{p u t}$ The response rate to non-elective demands for product $p$ with priority $u$ at period $t$

$E_{p t}$ : Amount of expiration of product $p$ at period $t$ (unit)

$O_{t}$ : Binary variable; 1 if the system replenishes any product units at period $t$; Otherwise, 0 
$E 1$ : Value of economic objective function (total system cost)

$H 1$ : Value of first health objective function (shortage)

$H 2$ : Value of second health objective function (substitution)

$H 3$ : Value of third health objective function (non-freshness)

H 4 : Value of fourth health objective function (non-elective demand)

\subsection{The objective functions}

There are five health-economic objective functions. One of them is an economic objective, and the other four are the objectives related to health equity. The proposed model objectives are as below:

\subsubsection{Economic objective}

The economic objective minimizes the total costs of inventory management. This function comprises four main parts: In the first part, the fixed and variable costs of the products, as well as purchasing costs, are calculated. In the second part, the purchasing cost of products using expedited service is computed to compensate for the shortage. In the third part, the holding cost is calculated. Finally, in the fourth part, the wastage/disposal cost of expired products is computed. Equation (6) shows the economic objective function and its different parts.

$$
\begin{aligned}
& \min E 1=\left[\sum_{t} F C ._{t}+\sum_{p} \sum_{t} P C_{p t} \cdot Q_{p t}\right]+\left[\sum_{p} \sum_{t} S C_{p t} X_{p t}\right]+\left[\sum_{p} \sum_{i} \sum_{t} H C_{p i} \cdot I_{p i t}\right] \\
& +\left[\sum_{p} \sum_{t} E C_{p} \cdot E_{p t}\right]
\end{aligned}
$$

\subsubsection{The first health objective}

The first health objective minimizes the inventory shortage. The shortage of medical products in healthcare systems has a direct impact on patients' health. Equation (7) shows how the first social objective function is calculated, by which the shortage is minimized.

$$
\min H 1=\sum_{p} \sum_{u} \sum_{t} \eta_{p u}\left(B_{p u t}-X_{p u t}\right)
$$

It should be pointed out that the value of different products for different ULs is considered to be stated in a standard manner. Also, the lost shortage may only happen in class 3 for the current problem.

\subsubsection{The second health objective}

The second health objective deals with minimizing the shortage-based substitution of products in demand with the rest of available products. Equation (8) shows the second social objective, by which the number of substitutions (specifically inappropriate substitution) is minimized. 


$$
\min H 2=\sum_{p} \sum_{i} \sum_{p^{\prime}, S^{p_{O}}{ }_{u}} \sum_{t}\left(1-\theta_{p p^{\prime} u}\right) F_{p i p^{\prime} u t}^{\prime^{\prime}}
$$

It should be mentioned that different products can be substituted with a specific substitution rate. The substitution objective function's sensitivity gets higher by decreasing the substitution rate, which leads to less substitution.

\subsubsection{The third health objective}

The third health objective is concerned with the freshness rate of items units assigned for demand satisfaction. This objective function is shown in Equation (9), in which demand satisfaction with non-fresh products is minimized. The freshness of each product and each demand priority has a specific value, where a product is considered as non-fresh if its age is more than $\frac{|I|}{2}$. Also, the relative importance coefficient of the freshness is multiplied by the degree of the non-freshness ( $\left.\frac{\left(i-\frac{|I|}{2}\right)}{|I|}\right)$ to increase the sensitivity to the non-fresh units.

$$
\min H 3=\left(\sum_{p} \sum_{i>\frac{I I}{2}} \sum_{u} \sum_{t} \frac{\pi_{p u}\left(i-\frac{|I|}{2}\right)}{|I|} F_{p i u t}+\sum_{p} \sum_{i} \sum_{p^{\prime}, S_{p_{O}} u} \sum_{t} \sum_{t} \frac{\pi_{p u}\left(i-\frac{|I|}{2}\right)}{|I|} F_{p i p u t}^{\prime}\right)
$$

\subsubsection{The fourth health objective}

The fourth health objective is stated in Equation (10), according to which the non-responding to non-elective demands is minimized. Note that $\pi_{p u}$ indicates the importance of each product for patients at each UL. This coefficient increases the response to emergency patients with high priority.

$$
\min H 4=\sum_{p} \sum_{u} \sum_{t} \eta_{p u}\left(1-Z_{p u t}\right) \cdot N D_{p u t}
$$

Five health-economic objectives are considered to model the current problem, including one economic and four health objectives dealing with healthcare equity. In classic approaches, mostly shortage is taken as a cost into account in the economic objective. However, due to the importance of shortage in this research, this objective is considered independently. 


\subsection{Model constraints}

Relations (11)-(34) address the proposed model constraints. Following each Constraint has been described in detail.

\subsubsection{Order quantity}

Constraint (11) shows the limited supply capacity. It states the order quantity can be equal to or less than the supply capacity. Moreover, Constraint (11) ensures that $O_{t}$ becomes 1 even if a single product unit is ordered from the blood center at day $t$ and 0 otherwise. Constraint (12) ensures that the replenishment can only occur during allowed review periods. Note that if $R=T$, Constraint (12) is not necessary.

Constraint (13) expresses that the total order quantity of the fresh units at period $t-1$ equals assigned units for demand satisfaction plus stock-on-hand at the end period $t$. Note that the received products by the blood bank at the beginning of the day $t$ are assumed to be fresh, and they are in the first period of their shelf-life. Moreover, the lead time to receive the order quantity from the supplier is considered one day.

$$
\begin{aligned}
& Q_{p t} \leq c_{p t} O_{t} ; \forall p \in P, t \in T \\
& O_{t}=0 ; \forall t \notin R \\
& Q_{p t-1}=Y_{p 1 t}+I_{p 1 t} ; \forall p \in P, t \in T
\end{aligned}
$$

\subsubsection{Inventory updates}

Constraint (14) shows that the stock-on-hand of a given item with remaining shelf-life of $i-1$ days at the end of the period $t-1$ equals the summation of the assigned units for demand satisfaction and stock-on-hand with shelf-life of $i$ days at the end of the period $t$ of the planning horizon.

$$
I_{p(i-1) t-1}=A_{p i t}+I_{p i t} ; \forall p \in P, i \in I-\{1\}, t \in T
$$

\subsubsection{Assigned/consumed inventory}

Constraint (15) indicates allocation and substitution of product $p$ units with shelf-life of $i$ days to meet the demands of class $u$ at day $i$ of their shelf-lives during the period $t$ of the planning horizon.

$$
A_{p i t}=\sum_{u} F_{p i u t}+\sum_{p^{\prime}, S_{O} P_{u}} \sum_{p^{\prime} p^{\prime} u t}^{\prime} ; \forall p \in P, i \in I, t \in T
$$




\subsubsection{Demand constraints}

Constraint (16) calculates the total satisfied demand of product $p$ at class $u$ and the period $t$ of the planning horizon. Also, the unfulfilled demand at class $u$ is considered as the shortage, namely $\mathrm{B}_{\text {put }}$, at period $t$, which might be compensated by shortage-based substitution, expedited-order,

or remained as lost depending on the demand's class which is predetermined and modeled by the following Constraints.

Constraint (17) shows how to satisfy the demand at class $u$ for product $p$ on the day $t$ of the planning horizon. In other words, Constraint (17) indicates how the demands for product $p$ are met by the same requested product and with other substitutable products if allowed.

$$
\begin{aligned}
& D_{p u t}=E D_{p u t}+Z_{p u t} . N D_{p u t}-B_{p u t} ; \forall p \in P, u \in U, t \in T \\
& \sum_{i} F_{p i u t}+\sum_{p^{\prime}, S_{O}^{p_{O}} i} \sum_{p^{\prime}{ }^{\prime} p u t}=D_{p u t} ; \forall p \in P, u \in U, t \in T
\end{aligned}
$$

\subsubsection{Shortage-based expedited order Policy}

Constraint (18) expresses that when demand at classes $u=1,2$ encounters the shortage for the product $p$, it must be satisfied through an expedited order policy. In this case, an extra order must be taken through expedited service. However, according to Constraint (19), it is unnecessary to compensate for the unfulfilled demand at class $u=3$. Note that the first health objective function (shortage) tends to compensate unfulfilled demand at class $u=3$ by ordering through expedited service.

$$
\begin{aligned}
& X_{p u t}=B_{p u t} ; \forall p \in P, u \in U-\{3\}, t \in T \\
& X_{p 3 t} \leq B_{p 3 t} ; \forall p \in P, t \in T
\end{aligned}
$$

\subsubsection{Expired/outdated items}

Constraint (20) calculates the number of expired units at the period $t$ of the planning horizon. Accordingly, the inventory of any unit at the end of the period $t-1$, which is at the last period of its shelf-life, is considered as the expired unit at the period $t$ of the planning horizon.

$$
E_{p t}=I_{p|I| t-1} ; \forall p \in P t \in T
$$

\subsubsection{Capacity constraints}

Constraints (21) and (22) address the total storage capacity of the system and the storage capacity limitation of each product at a period $t$ of the planning horizon. Note that the storage capacity of blood banks is limited because blood products need outstanding storage facilities. 


$$
\begin{aligned}
& \sum_{p} \sum_{i} m_{p} I_{p i t} \leq v \quad ; \forall t \in T \\
& \sum_{i} m_{p} I_{p i t} \leq m v_{p} \quad ; \forall p \in P \quad, t \in T
\end{aligned}
$$

\subsubsection{Substitution Policy}

Constraint (23) guarantees that the demand at $u=1$ will be satisfied. As long as there is a shortage of product $p$ for demand at class $u=1$, it is not possible to meet the demand of other classes. Similarly, Constraint (24) guarantees the priority of class $u=2$ over $u=3$. Note that if $\eta_{p 1}>\eta_{p 2}>\eta_{p 3}$, the second objective function satisfies Constraints (23) and (24) in optimality.

Constraint (25) shows how product substitution based on medical priorities can be performed at any ULs. For instance, if it is not possible to substitute any product at $u=1$, then, $\sum_{i} F_{\text {piput }}^{\prime}=0$. Note that $M$ is a very big number.

$$
\begin{aligned}
& B_{p l t}>0 \Rightarrow \sum_{u>1} F_{\text {piut }}+\sum_{p^{\prime}, S^{p} u>1} F_{\text {piput }}^{\prime}=0 ; \forall p \in P, i \in I, t \in T \\
& B_{p 2 t}>0 \Rightarrow \sum_{u>2} F_{\text {piut }}+\sum_{p^{\prime}, S^{p} u>2} F_{\text {piput }}^{\prime}=0 ; \forall p \in P, i \in I, t \in T \\
& \sum_{i} F_{p i p u t}^{\prime} \leq \delta_{p u} M ; \forall p \in P, p^{\prime}, S^{p p^{\prime}}, u \in U, t \in T
\end{aligned}
$$

\subsubsection{Issuance and allocation Policy}

Constraint (26) addresses the LEFO issuance policy for demand satisfaction at class $u=1$. In this way, the items of product $p$ with a shelf-life of $j>i$ days should not be used to satisfy the demand until the quantity of the product $p$ inventory with a shelf-life of $i$ days is exhausted. Note that the demands at class 1 must be satisfied with the freshest items.

$$
I_{p i t}>0 \Rightarrow \sum_{j>i} F_{p j u t}=0 ; \forall p \in P, i \in I, t \in T,[u=1]
$$

Constraint (27) shows Constraint (26) in a linear form. Note that in Constraint (27), $\ell_{\text {pit }}$ is a binary variable, which would be 1, if $I_{p i t}>0$. In Constraint (27), $M$ and eps are a positive big enough number and a very small number, respectively. 


$$
\left\{\begin{array}{c}
\ell_{p i t} \geq \frac{I_{p i t}}{M} ; \forall p \in P, i \in I, t \in T \\
\ell_{p i t} \leq 1+\frac{I_{p i t}-e p s}{M} ; \forall p \in P, i \in I, t \in T \\
\sum_{j>i} F_{p j u t} \leq\left(1-\ell_{p i t}\right) M ; \forall p \in P, i \in I, t \in T,[u=1] \\
\ell_{p i t} \in\{0,1\}
\end{array}\right.
$$

Constraints (28) and (29) address how FEFO and LEFO issuance policies are applied to satisfying the demand at $u=2$. Constraint (28) indicates that the fresh product units (with a shelf-life of fewer than $I / 2$ days) must be issued based on the FEFO policy. In this way, the product units with a shelf-life of $j<i$ days should not be used for demand satisfaction until the inventory with a shelf-life of $i$ days is exhausted. Also, Constraint (29) indicates that the fresh product units (with a shelf-life of more than $I / 2$ days) must be issued based on the LEFO policy. In this way, the product units with a shelf-life of $j>i$ days should not be used for demand satisfaction until the inventory with a shelf-life of $i$ days is exhausted. Note that for reducing the risk of lives, it is supposed to use the fresh items with LEFO policy and the ordinary product with FEFO policy for class 2 .

$$
\begin{gathered}
I_{p i t}>0 \Rightarrow \sum_{j<i} F_{p j u t}=0 ; \forall p \in P, i \leq \frac{|I|}{2}, t \in T,[u=2] \\
I_{p i t}>0 \Rightarrow \sum_{j>i} F_{p j u t}=0 ; \forall p \in P, i>\frac{|I|}{2}, t \in T,[u=2]
\end{gathered}
$$

The Constraints (30) and (31) show Constraints (28) and (29) in a linear form, respectively. Similarly, in Constraints (30) and (31), $\ell_{\text {pit }}$ is a binary variable, which would be 1 , if $I_{p i t}>0$. In these constraints, $M$ and are eps are respectively positive big enough and very small numbers.

$$
\left\{\begin{array}{c}
\ell_{p i t} \geq \frac{I_{p i t}}{M} ; \forall p \in P, i \in I, t \in T \\
\ell_{p i t} \leq 1+\frac{I_{p i t}-e p s}{M} ; \forall p \in P, i \in I, t \in T \\
\sum_{j<i} F_{p j u t} \leq\left(1-\ell_{p i t}\right) M ; \forall p \in P, i \leq \frac{|I|}{2}, t \in T,[u=2] \\
\ell_{p i t} \in\{0,1\}
\end{array}\right.
$$




$$
\left\{\begin{array}{c}
\ell_{p i t} \geq \frac{I_{p i t}}{M} ; \forall p \in P, i \in I, t \in T \\
\ell_{p i t} \leq 1+\frac{I_{p i t}-e p s}{M} ; \forall p \in P, i \in I, t \in T \\
\sum_{j>i} F_{p j u t} \leq\left(1-\ell_{p i t}\right) M ; \forall p \in P, i>\frac{|I|}{2}, t \in T,[u=2] \\
\ell_{p i t} \in\{0,1\}
\end{array}\right.
$$

As already pointed out, to meet patients' demand for products at class $u=3$ based on OP, the model itself decides which part of the inventory at the storage should be issued. Note that there is no difference in terms of freshness for demands in class 3. Nevertheless if the FEFO issuance policy is considered for demand assignment at class $u=3$. Constraint (32) would be applied. In this case, the production unit with a shelf-life of $j<i$ days should not be used for demand satisfaction until the product inventory with a shelf-life of $i$ days is exhausted.

$$
I_{p i t}>0 \Rightarrow \sum_{j<i} F_{p j u t}=0 ; \forall p \in P, i \in I, t \in T,[u=3]
$$

The Constraint (33) shows Constraint (32) in a linear form.

$$
\left\{\begin{array}{c}
\ell_{p i t} \geq \frac{I_{p i t}}{M} ; \forall p \in P, i \in I, t \in T \\
\ell_{p i t} \leq 1+\frac{I_{p i t}-e p s}{M} ; \forall p \in P, i \in I, t \in T \\
\sum_{j<i} F_{p j u t} \leq\left(1-\ell_{p i t}\right) M ; \forall p \in P, i \in I, t \in T,[u=3] \\
\ell_{p i t} \in\{0,1\}
\end{array}\right.
$$

\subsubsection{Non-negativity}

Constraint (34) represents non-negativity and binary restrictions in the model.

$$
\left\{\begin{array}{c}
O_{t}, \ell_{p i t} \in\{0,1\} \\
Q_{p t}, I_{p i t}, A_{p i t}, F_{p i u t}, F_{p i p u t}^{\prime}, G_{p u t}, D_{p u t}, B_{p u t}, W_{p u t}, E_{p t} \geq 0, G_{p u t} \leq 1
\end{array}\right.
$$




\subsection{Solution approach}

An extended GP approach is employed to make a trade-off between the objective functions of the proposed model. In this regard, the previously defined payoff matrix between health-economic objectives should be obtained in the beginning. The proposed model is first solved four times, each of which comprises only one of the considered objectives in terms of a single-objective problem. To find the appropriate interval corresponding to the $i^{t h}$ objective $(i=2, \ldots, n)$, the following optimization problem should be solved for each objective, $j=1,2, \ldots, n$ :

$$
\begin{gathered}
\operatorname{PayOff}_{j j}=\operatorname{Minf}_{j}(x) \\
x \in X
\end{gathered}
$$

Where, $x^{j,{ }^{*}}$ as the optimal solution and PayOff $f_{j j}=f_{j}\left(x^{j, *}\right)$ as the optimal value of the $j^{\text {th }}$ objective is saved. Then, the optimal value of the $i^{\text {th }}$ objective can be obtained by finding the optimal value of each objective $(j=1,2, \ldots, n ; j \neq i)$, as follows:

$$
\begin{gathered}
\operatorname{PayOff}_{i j}=\operatorname{Minf}_{i}(x) \\
f_{j}(x)=\text { PayOff }_{j j} \\
x \in X \\
j \neq i
\end{gathered}
$$

Where, $x^{i, j,{ }^{*}}$ and PayOff ${ }_{j j}=f_{i}\left(x^{i, j,{ }^{*}}\right)$ are the optimal solution and optimal value, belonging to the $i^{\text {th }}$ objective. Accordingly, the payoff matrix is obtained as follows:

$$
\text { PayOff }=\left[\text { PayOff }_{i j}\right]
$$

After determining the payoff matrix for each objective $(i=1,2, \ldots, n)$, the following items are defined:

- $\operatorname{Min}\left(f_{i}\right)=\operatorname{Min}_{j}\left\{\operatorname{PayOff}_{i j}\right\}=\operatorname{payOff}_{i i}$

- $\operatorname{Max}\left(f_{i}\right)=\operatorname{Max}_{j}\left\{\right.$ PayOff $\left._{i j}\right\}$

- $R\left(f_{i}\right)=\operatorname{Max}\left(f_{i}\right)-\operatorname{Min}\left(f_{i}\right)$

The $R\left(f_{i}\right)$ value is used to normalize the objectives in MODM objective function. Supposed that the following values are obtained using the objectives payoff matrices:

$\underline{F_{1}}:$ the minimum value of the first objective (optimal)

$\overline{F_{1}}$ : the minimum value of the first objective

$\underline{F_{2}}$ : the minimum value of the second objective (optimal)

$\overline{F_{2}}$ : the minimum value of the second objective 
$\underline{F_{3}}:$ the minimum value of the third objective (optimal)

$\overline{F_{3}}$ : the minimum value of the third objective

$F_{4}:$ the minimum value of the fourth objective (optimal)

$\overline{F_{4}}:$ the minimum value of the fourth objective

Next, using the GP approach, the MODM model can be stated in terms of a single-objective one, as Relation (38):

$$
\left\{\begin{array}{c}
\text { GP for MOMILP } \\
\text { min Z } \\
Z \geq \frac{Z^{E 1}-\underline{F_{1}}}{\overline{F_{1}}-\underline{F_{1}}} \\
Z \geq \frac{Z^{S 1}-\underline{F_{2}}}{\overline{F_{2}}-\underline{F_{2}}} \\
Z \geq \frac{Z^{S 2}-\underline{F_{3}}}{\overline{F_{3}}-\underline{F_{3}}} \\
Z \geq \frac{Z^{S 3}-\underline{F_{4}}}{\overline{F_{4}}-\underline{F_{4}}} \\
x \in X=\text { MOMILP Constraints }=\text { Eqs }(4-1)-\operatorname{Eqs}(4-29)
\end{array}\right.
$$

Note that the final multi-objective model is a MILP model by employing the GP approach. It could be solved using CPLEX Solver in GAMS.

\section{Case study}

This section presents a real case from Iran to validate the proposed mathematical model and evaluate its possible practicality. Iran is among the countries where blood donation is volunteerbased, so the amount of blood is strongly influenced by blood donors [32]. Hence, donors' low participation is always putting the country's BSC at risk of blood shortage. Previous studies on Iran's BSC and its challenges highlight the need to improve knowledge and activities related to blood management and its possible alternatives [15,18].

Iranian Blood Transfusion Organization (IBTO) is the only blood processor and supplier in Iran, namely, the blood center. IBTO is located in Tehran, the capital of Iran, and serves in-home hospitals directly. As the case study, a leading Cardiovascular hospital in Tehran is considered. The hospital is one of the biggest cardiovascular, medical, and research centers in west Asia, with a 24-hour emergency center. Therefore, the hospital encounters both elective and non-elective demands. 
Besides, the hospital offers preventive, diagnostic, and therapeutic services. In this direction, the hospital deals with patients at different levels of medical urgency. Also, heart transplants surgeries are performed in the cardiovascular center of this hospital. Most heart disease patients attend this hospital because they have a wide diversity and healthcare scope for patients. There are about 600 active beds in the hospital. There are about 600 active beds in the hospital. The hospital's blood bank's management team is responsible for controlling, policy-making, planning, and ordering the inventory of blood-base products required by the hospital's various departments to IBTO [35]. Moreover, the storage capacity of the blood bank and the supply capacity of the IBTO are limited. Figure 3 illustrates the relation between IBTO and the proposed blood bank.

The products under study are platelet-derived products with a shelf-life of 5 days and $\mathrm{ABO} / \mathrm{Rh}$ blood group types. The authors collected the required data through the previous study on the hospital blood bank's process model. In this regard, the blood bank's historical data, the IBTO statistical reports, and experts' opinions have been considered [35]. The data that has been used in this study were collected at the time of the outbreak of Coronavirus disease (Covid-19 pandemic). Tables A.1 to A.4 present the proposed model's parameter values for the system under study.

\section{Computational results and evaluation}

In this section, the inspired data from the predefined case study is applied to the problem. Then the validation of implementing the model is evaluated, and the results are drawn. In this research, a MOMILP model is proposed to handle the problem and then solved using a GP approach. The model is coded in GAMS (General Algebraic Modelling System) and solved by CPLEX solver on a personal computer with Intel Core i5 8400 processor, 8GB RAM, and SSD 120GB.

\subsection{Implementation and discussion}

In this part, the investigated problem is solved based on the approach described in Section 3. The validation of the proposed model is discussed through a real case study. The presented model's performance is compared with the single-objective models of each objective. In this regard, the proposed model is first solved five times, each of which comprises only one of the considered objectives in terms of a single-objective problem. Then, the multi-objective model is solved using a GP approach. Finally, the obtained results are discussed. The summary of the obtained results is inserted in Table 2 and compared in Figure 4 graphically.

Moreover, some sensitivity analyses are conducted on some of the problem's main parameters to assess the proposed model's performance. The obtained results help DMs find proper solutions for the problem.

In order to solve the problem using the GP approach, the payoff matrix should be first obtained. Next, as already explained in Section 4.4, the objective function's value is attained for the multiobjective model, using the payoff matrix. Table 2 addresses the obtained values. The SO and MO notations in the following Tables and Figures refer to the single- and multi-objective models, respectively. 
Figure 4 compares the results obtained from solving single- and multi-objective models. When only one objective is considered, the model gets the best possible solution for that objective. In this case, according to the results demonstrated in Table 2 and the plots in Figure 4, a proper solution is often not obtained for other objectives. The main challenge is making a trade-off among all objectives to get the best possible solution when several objectives are considered simultaneously. Figure 4 illustrates that the multi-objective model well-perform in this regard in comparison with other single-objective models.

Figure 5 indicates the solutions for order quantity and shortage obtain from single- and multiobjective models at a 30-day planning period. The results show that generally, larger order quantities are required to achieve less shortage. All unsatisfied demand in the single-objective model of shortage is compensated by imposing expedited-service costs through expedited-order to the IBTO. However, no expedited-order occurs for other single-objective models. In these cases, all shortage amounts are lost. The results show that the proposed multi-objective model's shortage amounts are significantly less than other single-objective models.

The single-objective model related to minimizing total system costs is an extended economic order quantity (EOQ) model. The EOQ model decreases the replenishment periods to a decrease in the system's total fixed-order cost. In this regard, the result of solving the single-objective economic model indicates that the number of replenishment periods over the planning horizon is equal to 17 . For other single-objective models related to health equity, it is equivalent to all allowed periods. However, for the proposed multi-objective model, replenishment is done in 18 periods to decrease the ordering fixed-cost. As the number of ordering periods decreases, the ordering quantities are relatively increased.

Figure 6 compares the rate of responding to non-elective demands for health-economic single- and multi-objective models. The results illustrate that the rate of single-objective related to nonelective demands is equal to $100 \%$. Hence, all non-elective demands are satisfied by this objective, and an optimum solution is obtained. Next, the proposed multi-objective model reaches the best solution for this health objective by meeting more than $70 \%$ of non-elective demands.

Moreover, for the first health-objective function, the response rate to non-elective demand is $46 \%$ which is better than the rest of single-objectives. This objective function is related to shortage minimization. Note that the shortage may occur for both elective and non-elective demands. In this case, the shortage objective function aims to respond to non-elective demand as much as possible.

Figure 7 indicates how the demands at different classes are fulfilled by the single- and multiobjective models. According to the problem's assumptions, substitution is not allowed for the first class's demands. However, for the other two classes, it depends on the medical preferences. In this case, the substitution single-objective model gets the optimum solution. Next, the results show that the proposed multi-objective model obtains the least substitution amounts compared to the other single-objective models. The worst-case belongs to the shortage objective. Note that the substitution rate increase to reduce the lost demands in the case of shortage. 
Figure 8 shows that most of the items assigned for demand satisfaction are nearly fresh for all health-economic single- and multi-objective models. The objectives related to the shortage and non-elective demands in the single-objective form satisfy a small part of demands with non-fresh items. The proposed model is designed in a way to consume the inventory before its expedition, if possible. Solving the proposed model verifies that the inventory is planned so that the wastage amount is optimal and equal to zero.

However, solving the economic single-objective model indicates that the freshness of items is less than the other models but still considered fresh. It is due to the nature of EOQ models. Note that in this case, the model reduces the number of replenishment periods to decrease the ordering fixedcost.

In this paper, the proposed multi-objective model is first solved using a GP approach with the same importance for all objectives. Next, the effect of objectives weight on the results is investigated to provide a comprehensive insight for decision-makers. Table 3 presents the effect of the healtheconomic objectives' weight on the obtained results.

Suppose that there is no fiscal constraint for the system and only the non-economic objectives be necessary for the system. In this case, Table 3 demonstrates that all the objectives related to health equity get near-optimal solutions. Nevertheless, the total system costs hugely increase. In this direction, the non-freshness objective has multi-optimal solutions.

According to Table 3, by optimizing the economic objective, the freshness objective also obtains an optimal solution. Suppose the weight of the economic objective function decreases. In that case, as the total system costs increase, the shortage decreases, and the rate of substitution increases. The weight reduction of the shortage objective function leads to a significant decrease in total system costs. By reducing the weight of the substitution objective, the total system costs and shortage amounts decrease significantly. The more important of the non-freshness objective has not a positive impact on the other health objective functions.

Most classic models focus only on cost and shortage minimization. In this case, most non-elective demands remain unsatisfied. Besides, the substitution rate gets very high. By reducing the weight of the economic objective, better solutions are obtained for the health equity objectives.

Figure 9 illustrates the effect of the expedited service cost (to compensate for the shortage) on the solution of normal order quantities, expedited orders quantities, shortage amounts, and lost demands. Increasing the expedited service cost affects the system's total costs related to inventory management and lost demands.

As discussed in the literature, the expedited service cost usually is high. As long as the expedited service cost is less than two times the variable purchasing cost, the model tends to meet the shortage of product units by receiving the expedited services. Henceforth, as the expedited service cost increases, the model prefers to order more quantities to face fewer shortage amounts and avoid using the expedited services as much as possible. Suppose the expedited service cost gets more than two times the variable purchasing cost. In that case, more quantities are ordered, and the amounts of lost demand and shortage overlap. 
The proposed model is designed so that the products' availability for class 1 and class 2 is of great importance, respectively. If the inventory system at these two levels faces a shortage in demand satisfaction, the required item units would be supplied immediately by imposing the cost of expedited services. In this case, the Constraints (4-18) and (4-19) are relaxed; only the value of $\eta_{p u}$ affects the importance of product availability and the shortage of the two first levels of medical urgency. Moreover, the obtained solution does not necessarily lead to satisfying the unfulfilled demand through expedited services when the system faces a shortage.

The effect of the value of $\eta_{p 3}$ parameter, on the solution of the proposed multi-objective model is investigated, and the obtained results are reported in Table 4 . The results show that the amounts of wastage and expedited order are zero in all cases.

Figure 10 demonstrates the effect of purchasing fixed-cost on the order quantity and shortage by solving the proposed multi-objective model. It also indicates the relation of replenishment periods and the order quantity by increasing the purchasing fixed-cost. The higher the purchasing fixedcost, the lower the number of ordering periods, and the higher the order quantity in each period. The higher amount of order quantities straightly impacts the shortage. The higher the order quantity leads to a lower shortage. Up to $F C=50000$ the number of replenishment periods is gradually decreased. Similarly, the order quantity is gradually increased, and the amount of shortage is decreased. After $F C=50000$ the replenishment periods remain steady. Nevertheless, after this point, the order quantities instantly increase with a steep slope. The amount of shortage has the opposite behavior to the order quantity.

\subsection{Managerial insights}

This paper proposes a comprehensive inventory management model for hospital blood banks to select logistical tactics, inventory levels, and supply chain designs. The model has been examined in a real-case to evaluate its validity. Also, some sensitivity analyses are conducted to investigate the model's performance to provide a wide view for decision-makers. Various insights are provided below to understand the benefits of the proposed model and its obtained solution to help managers make appropriate decisions. The most important insights of this paper are as follows:

- Making appropriate inventory management decisions when the healthcare system pursues multiple and contradictory health-economic objectives related to financial resources and health equity;

- Applying the concepts of health equity in blood inventory management pragmatically;

- Considering the medical priorities of patients in meeting the blood demands and adopting appropriate issuance and allocation policies in this regard;

- Making a trade-off between the total costs of the system and equity in health by eliminating unnecessary operational costs without weakening the level of service delivery in the system where all health and economic objectives are influential; 
- Providing an efficient blood inventory management model to improve medical service, expedite treatments, and avoid the risk of lives.

- Making optimal decisions about the order quantities and replenishment periods for a real healthcare system so that the least wastage and shortage occurs in the system;

- Adopting an appropriate heuristic policy to deal with non-elective and emergency demands;

- Preparing the data needed for logistic plans and operational infrastructure for adopting an effective logistical strategy for blood products.

\section{Conclusions and future studies}

The blood banks' ultimate purpose is to provide blood products in the required quantity and right time so that minimum shortage and wastage occur. Making a trade-off between system costs and equity objectives without weakening the service quality is one of the most challenging healthcare issues. In this regard, this investigation presents a practical demand-oriented health-economic MOMILP model for blood inventory management of a healthcare system. Besides, an effective hybrid heuristic is extended to issue and allocate the blood for demands at different urgent classes optimally and minimize product substitution, shortage, and wastage. Also, fresher inventory items are used for patient treatment. This paper is the first operational research study investigating issues related to health equity in order to provide a desirable level of accessibility of medical services in the aspect of inventory management. In this regard, some practical strategies and policies have been proposed which can be beneficial for handling the shortage of substances of human origin and critical medicines during the Covid-19 pandemic.

An extended GP technique is utilized to obtain the optimal solution. Three demand classes are considered according to medical priorities to implicate equity in health pragmatically. Also, the proposed model considers non-elective demands and offers a way to deal with emergency conditions. The presented model is implemented for a real-case study in Iran. Some sensitivity analyses are conducted on the main parameters to evaluate the model performance and provide practical insight for decision-makers.

The results illustrate that the proposed health-economic trade-off is well-performed. The results show that if each objective is considered independently in a single-objective format, the optimal solution will be obtained. Nevertheless, for other objectives, the fitness of the solution is mostly not appropriate. According to the results, the proposed multi-objective model gets the best solution when all objectives are considered together. Hence, the proposed model gives a proper solution to meet demands with different priorities.

This investigation helps the managers to consider health equity along with economic objectives. The model contributes to achieving inventory management strategies that are beneficial to meet the system's demand more desirably and prevent blood loss as much as possible. Our proposed policy's goal is to satisfy the patient's demand with minimum mismatches and treatment risks. The presented model helps the managers to act decisively for blood inventories and increase the medical service level to the desired level. The findings are helpful in selecting logistical tactics, inventory policies, and supply chain designs. The presented model can also be used to design and improve blood decision support systems. 
In recent years, BSC has been exposed to a decline in blood donors due to the COVID-19 pandemic, resulting in a more significant shortage of blood resources. This issue adds to the importance of applying equitable policies in blood allocation. Future research can focus on blood inventory management strategies and practical implementation of health equity during the pandemics. To do so, one can consider the whole BSC network or different hierarchical levels and use different modeling approaches and solution techniques. Integrating the concept of resilience into the solution methodology's design by testing the proposed model's behavior on unexpected disruptions in blood banks could be a future stream. In this regard, developing the proposed model for handling real-world uncertainties could be interesting for future research. Designing and optimizing a sustainable integrated BSC network considering health equity can be another research topic. Moreover, human origin substances other than the blood are susceptible due to supply constraints and high demand levels. Extending and evaluating the proposed model for such products is highly encouraged.

The take-home message is to classifying demand priorities for the limited blood resources to minimize the risk of life and promote health services. Those responsible for the logistics and inventory of a blood bank expected to ensure the correct replenishment of the blood products, when needed, with the quality and quantity intended to provide healthcare service. Hence, they are looking for practical strategies to avoid the risk of lives besides ensuring that the whole process is carried out in the most eff ective and efficient way. They also need to look for opportunities to improve inventory management processes to reduce costs and improve medical service continually. In this regard, managing inventory is imperative to identify and correct the logistic deficiencies.

\section{Appendix. Parameters' value}

\section{References}

1. Braveman, P., "Health disparities and health equity: concepts and measurement", Annu. Rev. Public Heal., 27, pp. 167-194 (2006).

2. Mousazadeh, M., Torabi, S. A., Pishvaee, M. S., et al., "Accessible, stable, and equitable health service network redesign: A robust mixed possibilistic-flexible approach", Transp. Res. Part E Logist. Transp. Rev., 111, pp. 113-129 (2018).

3. van Roode, T., Pauly, B. M., Marcellus, L., et al., "Values are not enough: qualitative study identifying critical elements for prioritization of health equity in health systems", Int. J. Equity Health, 19(1), pp. 1-13 (2020).

4. Culyer, A. J., "Equity-some theory and its policy implications", J. Med. Ethics, 27(4), pp. 275-283 (2001).

5. Braveman, P. and Gruskin, S., "Defining equity in health", J. Epidemiol. Community Heal., 57(4), pp. 254-258 (2003).

6. Beier, F. J., "The management of the supply chain for hospital pharmacies: a focus on inventory management practices", J. Bus. Logist., 16(2), p. 153 (1995). 
7. Liu, W., Ke, G. Y., Chen, J., et al., "Scheduling the distribution of blood products: A vendormanaged inventory routing approach", Transp. Res. Part E Logist. Transp. Rev., 140, p. 101964 (2020).

8. Civelek, I., Karaesmen, I., and Scheller-Wolf, A., "Blood platelet inventory management with protection levels", Eur. J. Oper. Res., 243(3), pp. 826-838 (2015).

9. Derikvand, H., Hajimolana, S. M., Jabbarzadeh, A., et al., "A robust stochastic bi-objective model for blood inventory-distribution management in a blood supply chain", Eur. J. Ind. Eng., 14(3), pp. 369-403 (2020).

10. Cheraghi, S. and Hosseini-Motlagh, S.-M., "Responsive and reliable injured-oriented blood supply chain for disaster relief: a real case study", Ann. Oper. Res., pp. 1-39 (2018).

11. Pulok, M. H., van Gool, K., Hajizadeh, M., et al., "Measuring horizontal inequity in healthcare utilisation: A review of methodological developments and debates", Eur. J. Heal. Econ., 21(2), pp. 171-180 (2020).

12. Dahlgren, G., "Efficient equity-oriented strategies for health", Bull. World Health Organ., 78, pp. 79-81 (2000).

13. Saha, E. and Ray, P. K., "Inventory Management and Analysis of Pharmaceuticals in a Healthcare System”, In Healthcare Systems Management: Methodologies and Applications, Springer, pp. 7195 (2018).

14. Wilson, K. and Hébert, P. C., "The challenge of an increasingly expensive blood system", Cmaj, 168(9), pp. 1149-1150 (2003).

15. Hosseini-Motlagh, S.-M., Samani, M. R. G., and Homaei, S., "Blood supply chain management: robust optimization, disruption risk, and blood group compatibility (a real-life case)", J. Ambient Intell. Humaniz. Comput., 11(3), pp. 1085-1104 (2020).

16. Najafi, M., Ahmadi, A., and Zolfagharinia, H., "Blood inventory management in hospitals: Considering supply and demand uncertainty and blood transshipment possibility", Oper. Res. Heal. Care, 15, pp. 43-56 (2017).

17. Larimi, N. G., Yaghoubi, S., and Hosseini-Motlagh, S.-M., "Itemized platelet supply chain with lateral transshipment under uncertainty evaluating inappropriate output in laboratories", Socioecon. Plann. Sci., 68, p. 100697 (2019).

18. Hosseini-Motlagh, S.-M., Samani, M. R. G., and Cheraghi, S., "Robust and stable flexible blood supply chain network design under motivational initiatives", Socioecon. Plann. Sci., 70, p. 100725 (2020).

19. Ezugwu, A. E., Olusanya, M. O., and Govender, P., "Mathematical model formulation and hybrid metaheuristic optimization approach for near-optimal blood assignment in a blood bank system", Expert Syst. Appl., 137, pp. 74-99 (2019).

20. Rajendran, S. and Ravindran, A. R., "Inventory management of platelets along blood supply chain to minimize wastage and shortage”, Comput. Ind. Eng., 130, pp. 714-730 (2019).

21. Leaven, L., Ahmmad, K., and Peebles, D., "Inventory management applications for healthcare supply chains”, Int. J. Supply Chain Manag, 6, pp. 1-7 (2017).

22. Dillon, M., Oliveira, F., and Abbasi, B., "A two-stage stochastic programming model for inventory management in the blood supply chain”, Int. J. Prod. Econ., 187, pp. 27-41 (2017). 
23. Rajendran, S. and Srinivas, S., "Hybrid ordering policies for platelet inventory management under demand uncertainty", IISE Trans. Healthc. Syst. Eng., 10(2), pp. 113-126 (2020).

24. Dehghani, M. and Abbasi, B., "An age-based lateral-transshipment policy for perishable items", Int. J. Prod. Econ., 198, pp. 93-103 (2018).

25. Clay, N. M., Abbasi, B., Eberhard, A., et al., "On the volatility of blood inventories", Int. Trans. Oper. Res., 25(1), pp. 215-242 (2018).

26. Rajendran, S. and Ravindran, A. R., "Platelet ordering policies at hospitals using stochastic integer programming model and heuristic approaches to reduce wastage", Comput. Ind. Eng., 110, pp. 151-164 (2017).

27. Najafi, M., Ahmadi, A., and Zolfagharinia, H., "Blood inventory management in hospitals: Considering supply and demand uncertainty and blood transshipment possibility", Oper. Res. Heal. Care, 15, pp. 43-56 (2017).

28. Hosseinifard, Z. and Abbasi, B., "The inventory centralization impacts on sustainability of the blood supply chain”, Comput. Oper. Res., 89, pp. 206-212 (2018).

29. Abbasi, B. and Hosseinifard, S. Z., "On the issuing policies for perishable items such as red blood cells and platelets in blood service”, Decis. Sci., 45(5), pp. 995-1020 (2014).

30. Abdulwahab, U. and Wahab, M. I. M., "Approximate dynamic programming modeling for a typical blood platelet bank", Comput. Ind. Eng., 78, pp. 259-270 (2014).

31. Pirabán, A., Guerrero, W. J., and Labadie, N., "Survey on blood supply chain management: Models and methods", Comput. Oper. Res., 112, p. 104756 (2019).

32. Hosseini-Motlagh, S.-M., Samani, M. R. G., and Homaei, S., "Toward a coordination of inventory and distribution schedules for blood in disasters", Socioecon. Plann. Sci., p. 100897 (2020).

33. Fontaine, M. J., Chung, Y. T., Rogers, W. M., et al., "Improving platelet supply chains through collaborations between blood centers and transfusion services", Transfusion, 49(10), pp. 2040 2047 (2009).

34. Behera, B. K. and Varma, A., "Microbial Products Supply Chain”, In Microbial Biomass Process Technologies and Management, Springer, pp. 215-255 (2017).

35. Sohrabi, M., Zandieh, M., and Nadjafi, B. A., "Dynamic Demand-Centered Process-Oriented Data Model for Inventory Management of Hemovigilance Systems", Healthc. Inform. Res., 27(1), pp. 73-81 (2021).

36. Manopiniwes, W. and Irohara, T. "Stochastic optimisation model for integrated decisions on relief supply chains: preparedness for disaster response", Int. J. Prod. Res., 55(4), pp. 979-996 (2017).

37. Gunantara, N., "A review of multi-objective optimization: Methods and its applications", Cogent Eng., 5(1), p. 1502242 (2018).

38. Mosadegh, H., Khakbazan, E., Salmasnia, A., et al., "A fuzzy multi-objective goal programming model for solving an aggregate production planning problem with uncertainty", Int. J. Inf. Decis. Sci., 9(2), pp. 97-115 (2017).

39. Jones, D. and Tamiz, M., “A review of goal programming”, In Multiple Criteria Decision Analysis, Springer, pp. 903-926 (2016).

40. Coello, C. A. C., Lamont, G. B., and Van Veldhuizen, D. A., Evolutionary Algorithms for Solving 
Multi-Objective Problems, Springer (2007).

41. Osorio, A. F., Brailsford, S. C., Smith, H. K., et al., "Designing the blood supply chain: how much, how and where?", Vox Sang., 113(8), pp. 760-769 (2018).

42. Sarhangian, V., Abouee-Mehrizi, H., Baron, O., et al., "Threshold-based allocation policies for inventory management of red blood cells", Manuf. Serv. Oper. Manag., 20(2), pp. 347-362 (2018).

43. Samani, M. R. G., Hosseini-Motlagh, S.-M., and Ghannadpour, S. F., "A multilateral perspective towards blood network design in an uncertain environment: Methodology and implementation", Comput. Ind. Eng., 130, pp. 450-471 (2019).

44. Uthayakumar, R. and Karuppasamy, S. K., "An EOQ model for deteriorating items with different types of time-varying demand in healthcare industries", J. Anal., 27(1), pp. 3-18 (2019).

45. Paul, A., Rajapakshe, T., and Mallik, S., "Socially optimal contracting between a regional blood bank and hospitals", Prod. Oper. Manag., 28(4), pp. 908-932 (2019).

46. Srinivas, S. and Rajendran, C., "Community detection and influential node identification in complex networks using mathematical programming”, Expert Syst. Appl., 135, pp. 296-312 (2019).

47. Attari, M. Y. N. and Abdoli, N., "Reducing wastages and shortages of blood products at hospitals with considering ABO/Rh (D) compatibility", J. Ind. Eng. Decis. Mak., 1(1), pp. 24-48 (2020).

\section{Bibliography}

Mahnaz Sohrabi is a Ph.D. candidate in Industrial Engineering at the Islamic Azad University, Qazvin Branch, Iran. She accomplished her M.Sc. in Industrial Engineering in 2014. Her research interests include applied operations research, supply chain modeling, inventory and production planning, project scheduling, and optimization under uncertainty in real-world systems. E-mail address: $\underline{\text { m.sohrabi@,qiau.ac.ir. }}$

Mostafa Zandieh accomplished his B.Sc. in industrial engineering at Amirkabir University of Technology, Tehran, Iran (1994-1998), and M.Sc. in industrial engineering at Sharif University of Technology, Tehran, Iran (1998-2000). He obtained his Ph.D. in industrial engineering from Amirkabir University of Technology, Tehran, Iran (2000-2006). Currently, he is a professor at the Industrial Management and Information Technology Department, Shahid Beheshti University, Tehran, Iran. His research interests are production planning and scheduling, financial engineering, quality engineering, applied operations research, simulation, and artificial intelligence techniques in manufacturing systems design. E-mail address: $m$ zandieh@sbu.ac.ir.

Behrouz Afshar-Nadjafi received his Ph.D. degree in Industrial Engineering from the Sharif University of Technology in 2008 and is currently an Associate Professor and faculty member in the Department of Industrial Engineering at the Islamic Azad University, Qazvin Branch, in Iran. His research interests include project scheduling, inventory, and production planning. More specifically, 
he is working on modeling and efficient solution methods regarding optimization problems in reality. E-mail: afsharnb@alum.sharif.edu.

\section{Figure and table captions}

Fig. 1. Specifications of demand classes.

Fig. 2. The inventory flows in the proposed system.

Fig. 3. The case study definition.

Fig. 4. Comparison of obtained values for five health-economic objectives by solving single-objective models.

Fig. 5. Obtained solutions for order quantity and shortage by solving single- and multi-objective models.

Fig. 6. The response rate to non-elective demand for single- and multi-objective models.

Fig. 7. How to assign inventory to different levels of demand urgency for single- and multi-objective models.

Fig. 8. The freshness of the assigned products for single- and multi-objective models.

Fig. 9. Results of changing expedited-order cost (which is considered as a proportion of variable purchasing cost) on normal/expedited order quantity, shortage, and lost demand.

Fig. 10. Results of changing purchasing fixed-cost on order quantity, shortage, and replenishment periods.

Table 1. Summary of the most related papers in the field of inventory management of medical products in recent publications.

Table 2. Result summary of the single- and multi-objective models.

Table 3. Results of changing the health-economic objectives' weights on the optimal solution of the multiobjective model.

Table 4. Results of changing the product availability importance $\left(\eta_{p u}\right)$ on the solution of the multi-objective model during the planning horizon.

Table A.1. Substitution rate of product $\mathrm{p}$ with product p' for demand at class 3 .

Table A.2. Variable purchasing costs.

Table A.3. Parameters' value of the proposed model.

Table A.4. Products substitution allowance based on medical priorities. 


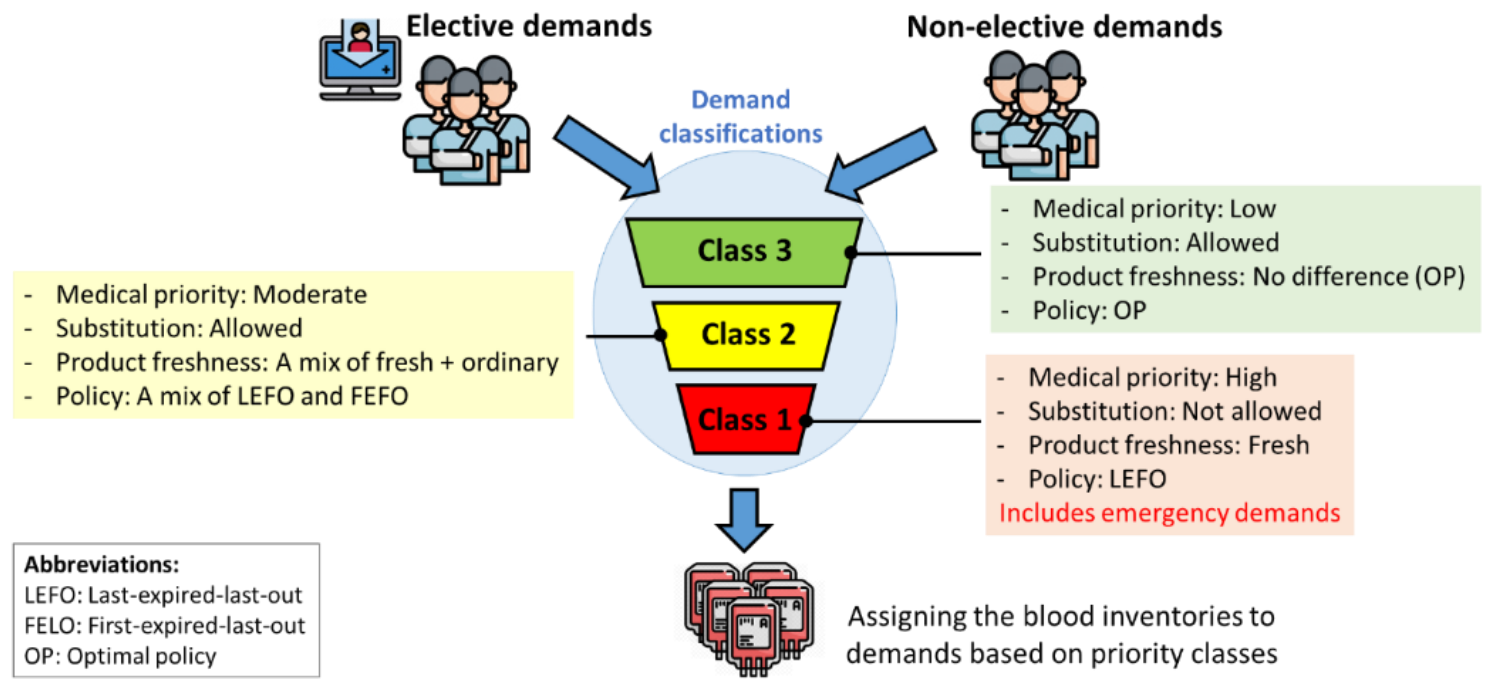

Fig. 1

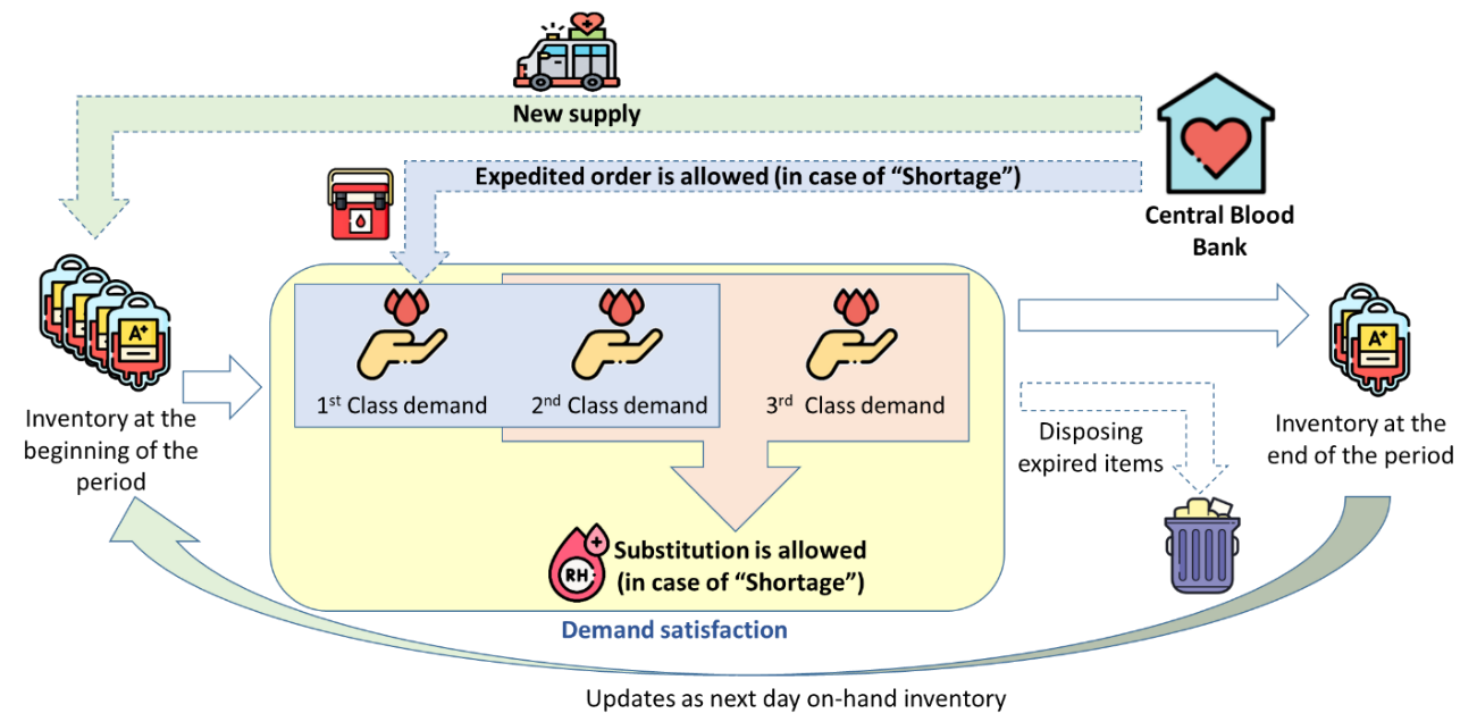

Fig. 2 


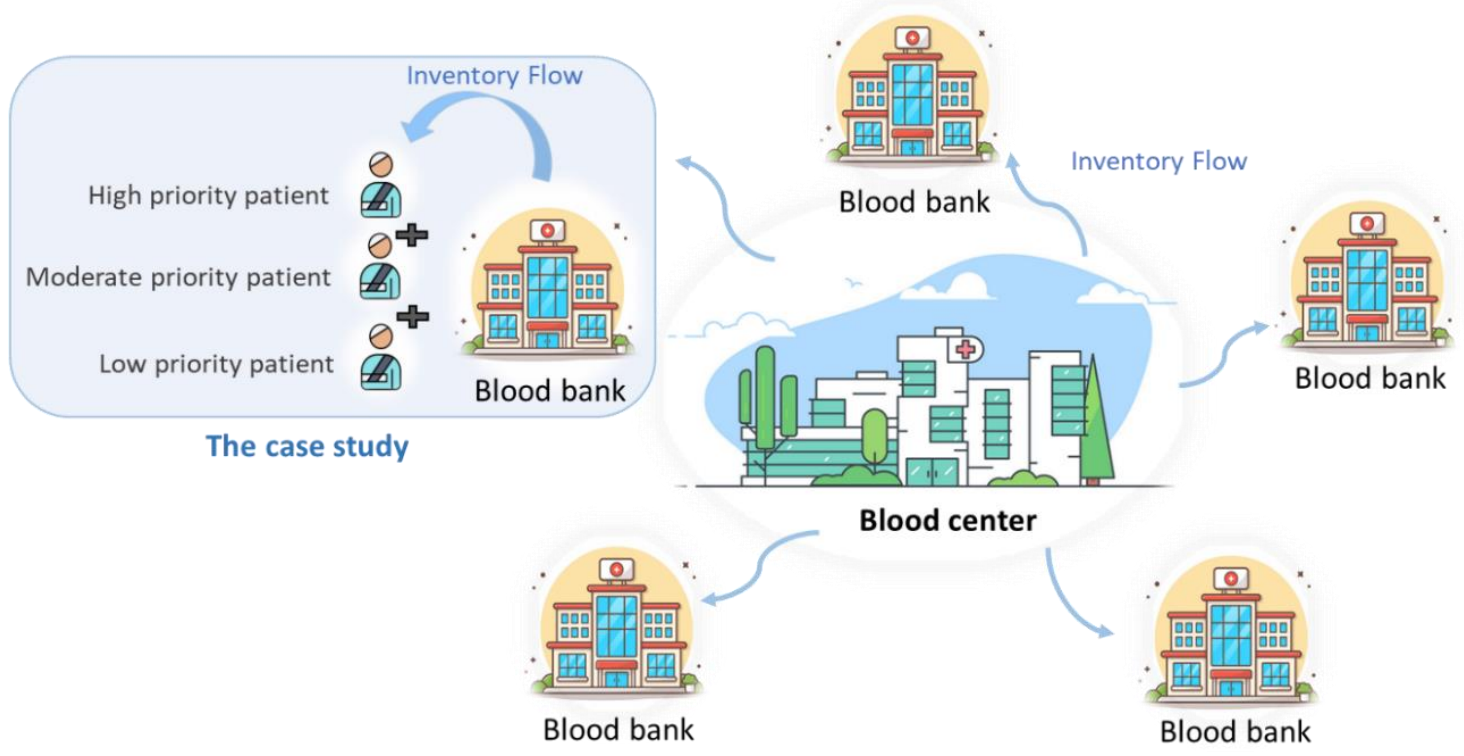

Fig. 3

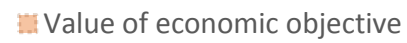

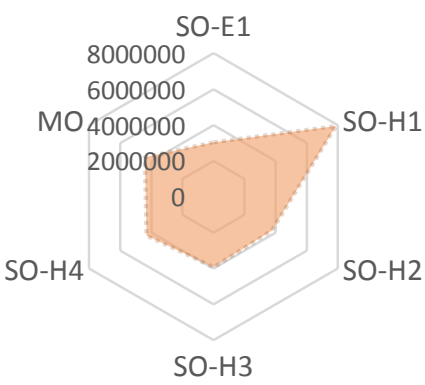

Value of shortage objective

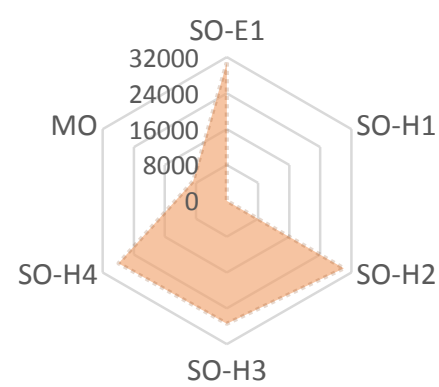

Value of substitution objective

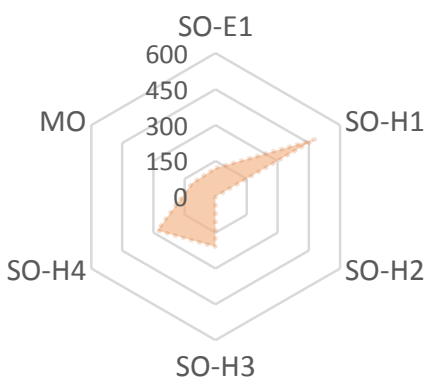

Value of non-freshness objective

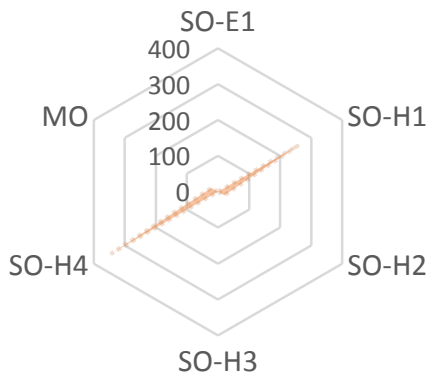

蕒 Value of non-elective demand objective

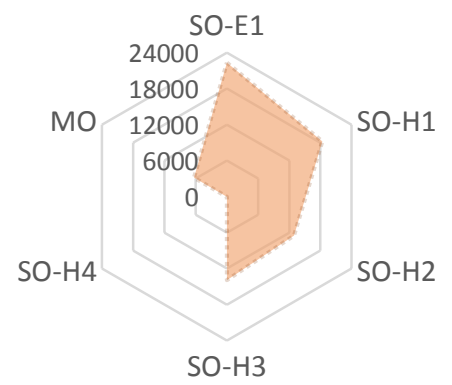

Fig. 4 


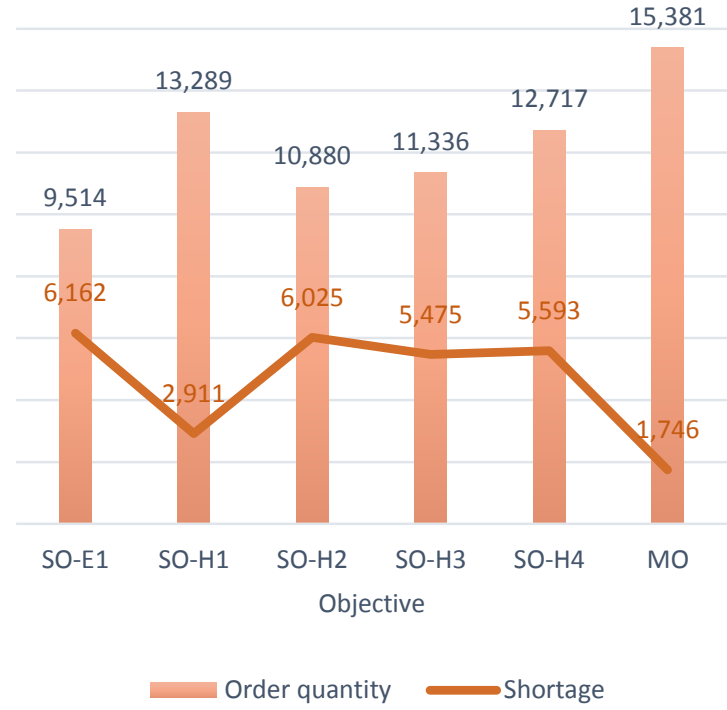

Fig. 5

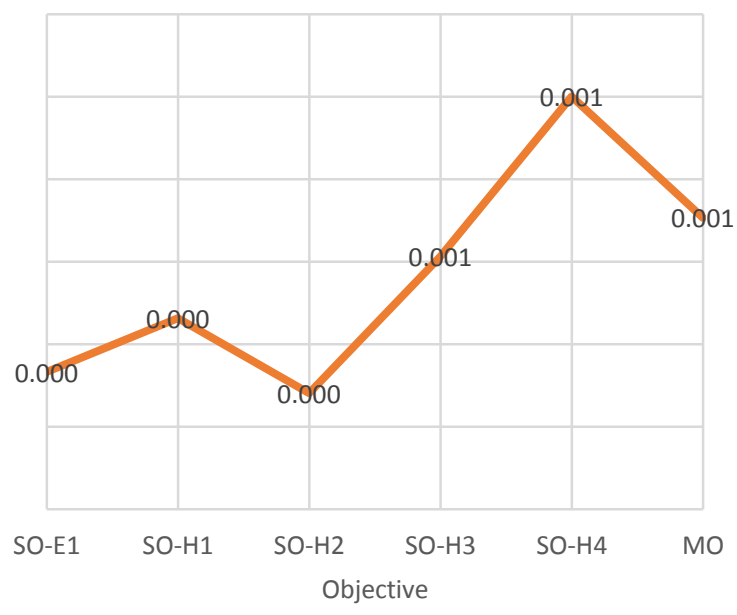

Response rate to non-elective demand

Fig. 6 


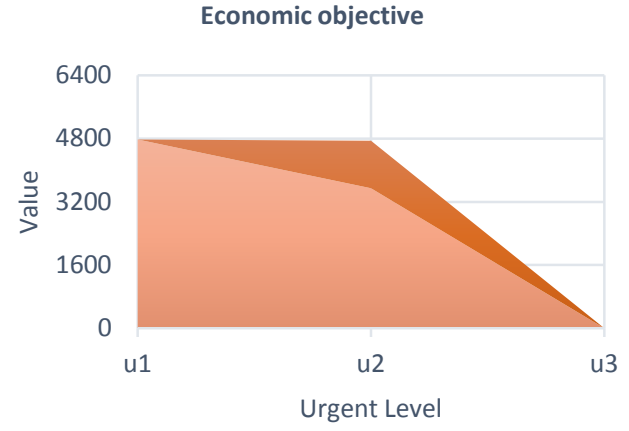

Normal assignment Substitution

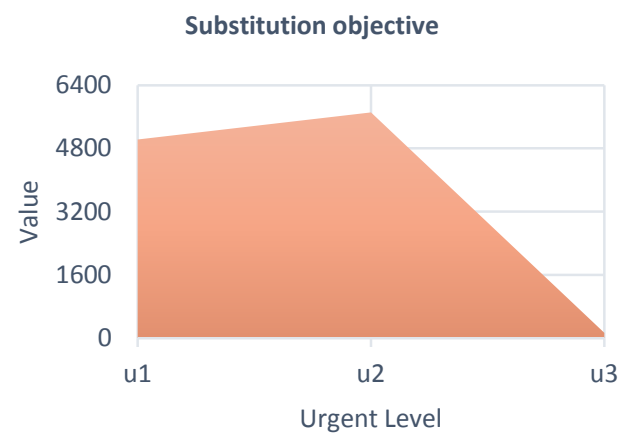

Normal assignment Substitution

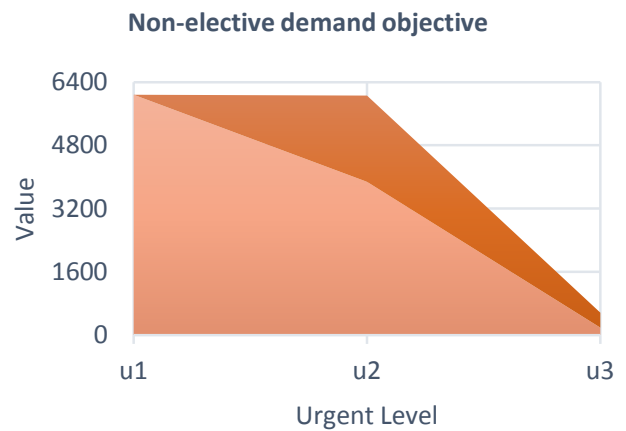

Normal assignment $\square$ Substitution

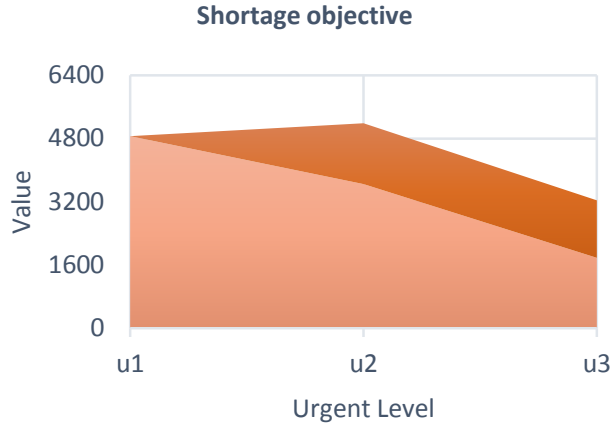

Normal assignment Substitution

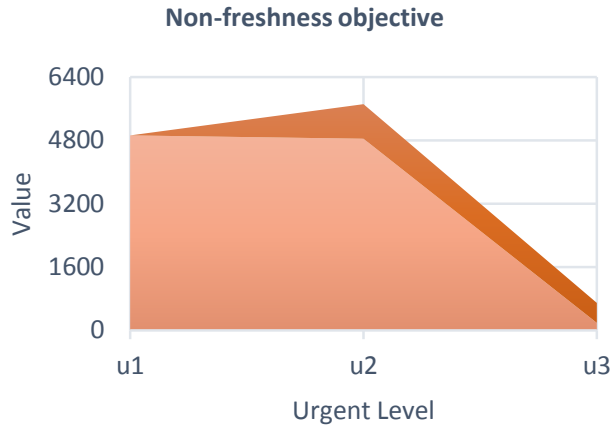

Normal assignment Substitution

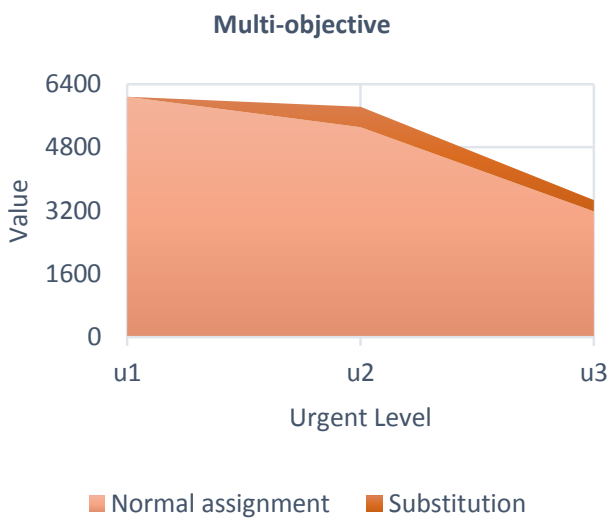

Fig. 7 


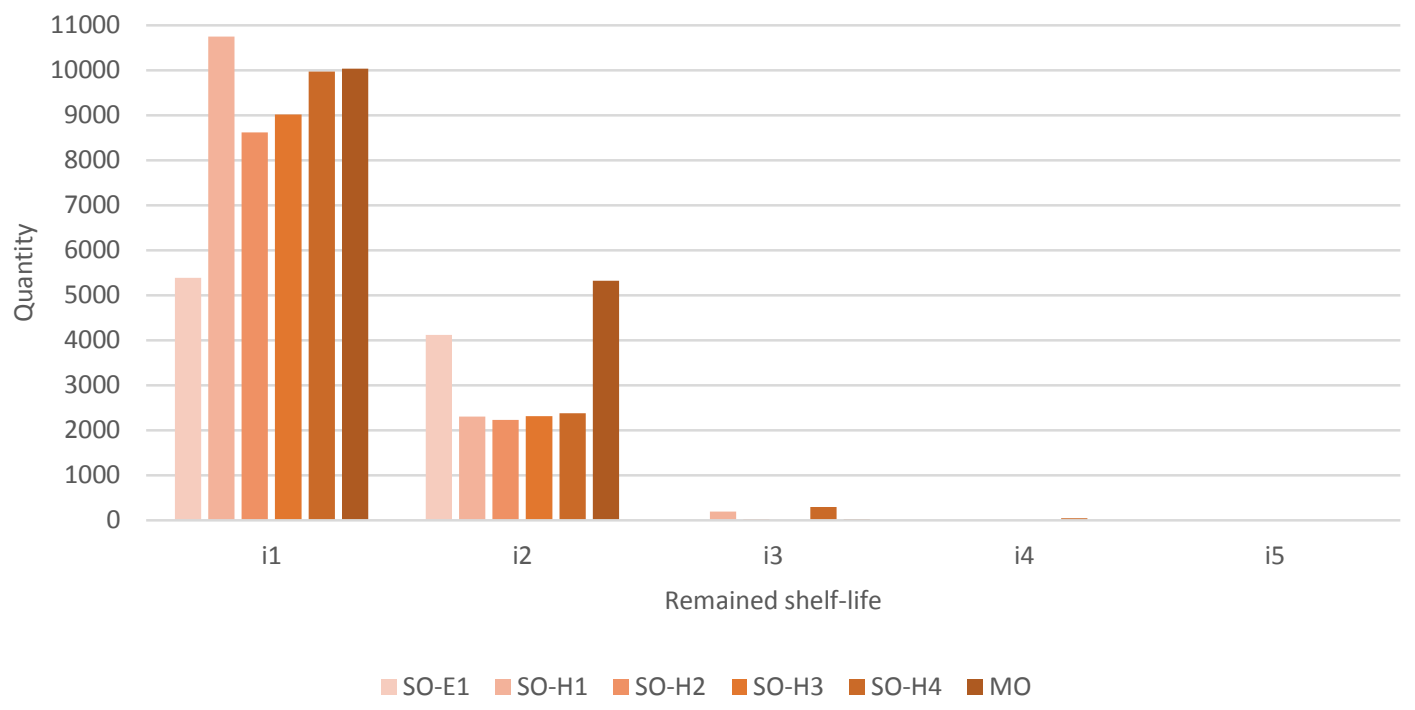

Fig. 8

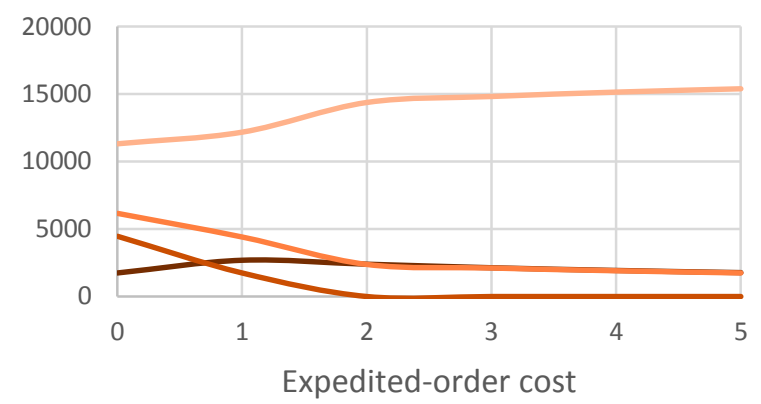

- Lost demand

Expedited-order quantity

Shortage

Normal-order quantity

Fig. 9 
The relation between shortage and order quantity

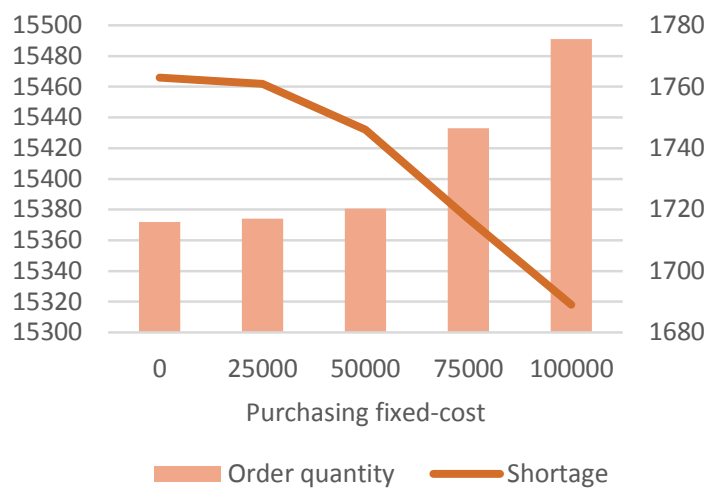

Order quantity along with replenishment periods

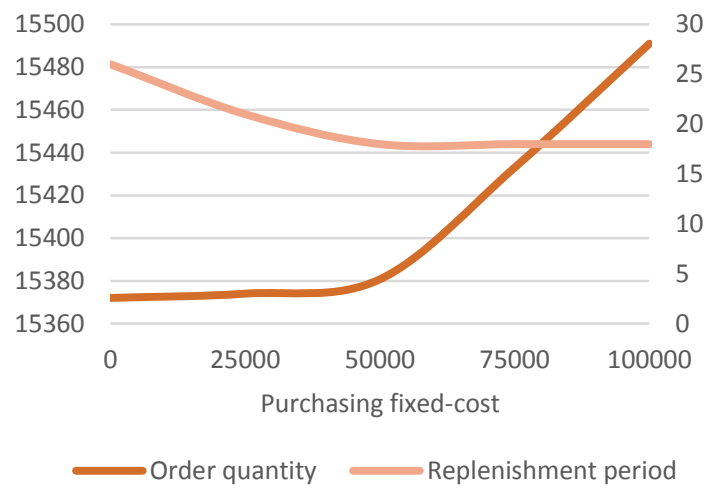

Fig. 10 


\begin{tabular}{|c|c|c|c|c|c|c|c|c|c|c|c|c|c|}
\hline Authors & $\begin{array}{l}\text { No. of } \\
\text { Products }\end{array}$ & Obj. & $\begin{array}{c}\text { Ordering } \\
\text { Policy }\end{array}$ & $\begin{array}{c}\text { Issuing } \\
\text { Policy }\end{array}$ & $\begin{array}{c}\text { Model. } \\
\text { approach }\end{array}$ & $\begin{array}{l}\text { Solution } \\
\text { Method } \\
\end{array}$ & $\begin{array}{c}\text { Demand } \\
\text { Differentiations } \\
\end{array}$ & $\begin{array}{c}\text { Capacity } \\
\text { Constraints }\end{array}$ & Substitution & $\begin{array}{c}\text { Emergency } \\
\text { condition }\end{array}$ & $\begin{array}{c}\text { Expedited } \\
\text { orders }\end{array}$ & Equity & $\begin{array}{l}\text { Case } \\
\text { Study }\end{array}$ \\
\hline $\begin{array}{l}\text { Rajendran \& Ravindran } \\
\text { (2017) [26] }\end{array}$ & SP & SO & $\begin{array}{l}(\mathrm{s}, \mathrm{S}), \mathrm{BaS}, \\
\text { MBS,WM } \\
\text { V,LVM }\end{array}$ & FIFO & MILP & $\mathrm{H}$ & & & & & $*$ & & $\mathrm{~N}$ \\
\hline Dillon et al. (2017) [22] & MP & SO & $(\mathrm{R}, \mathrm{S})$ & & MILP & EX & & & $*$ & & $*$ & & $\mathrm{Y}$ \\
\hline $\begin{array}{l}\text { Dehghani and Abbasi } \\
\text { (2018) [24] }\end{array}$ & SP & SO & $(\mathrm{S}-1, \mathrm{~S})$ & FIFO & NLP & $\mathrm{H}$ & & & & & $*$ & & Y \\
\hline $\begin{array}{l}\text { Hosseinifard and Abbasi } \\
\text { (2018) [28] }\end{array}$ & SP & SO & $(\mathrm{S}-1, \mathrm{~S})$ & FIFO & MILP & EX & & St & & & & & $\mathrm{N}$ \\
\hline Osorio et al. (2018) [41] & MP & MO & $\mathrm{OP}$ & & MILP & $\mathrm{H}$ & & $S$ & & & & & $\mathrm{Y}$ \\
\hline $\begin{array}{l}\text { Sarhangian et al. (2018) } \\
\text { [42] }\end{array}$ & SP & SO & OP & FIFO & NLP & SIM & & & & & & & $\mathrm{N}$ \\
\hline $\begin{array}{l}\text { Samani and Hosseini- } \\
\text { Motlagh (2019) [43] }\end{array}$ & MP & MO & $\mathrm{OP}$ & & MILP & EX & & $\mathrm{S}, \mathrm{St}$ & & & & & Y \\
\hline $\begin{array}{l}\text { Karuppasamy and } \\
\text { Uthayakumar (2019) } \\
\text { [44] }\end{array}$ & SP & SO & EOQ & & NLP & $\mathrm{AT}$ & & & & & & & Y \\
\hline Paul et al. (2019) [45] & SP & SO & $\mathrm{OP}$ & & MILP & EX & & & & & $*$ & & $\mathrm{~N}$ \\
\hline $\begin{array}{l}\text { Ezugwu and Govender } \\
\text { (2019) [19] }\end{array}$ & MP & SO & $\mathrm{OP}$ & FIFO & MILP & MH & & & & & & & $\mathrm{N}$ \\
\hline $\begin{array}{l}\text { Rajendran \& Ravindran } \\
\text { (2019) [20] }\end{array}$ & SP & SO & $(\mathrm{s}, \mathrm{S}, \alpha, \mathrm{Q})$ & FIFO & MILP & MH & & & & & $*$ & & Y \\
\hline Larimi et al. (2019) [17] & MP & MO & & FIFO & MILP & EX & SL & $\mathrm{S}, \mathrm{St}$ & & & & & Y \\
\hline $\begin{array}{l}\text { Srinivas \& Ravindran } \\
\text { (2019) [46] }\end{array}$ & SP & SO & $(\mathrm{s}, \mathrm{S}, \beta, \mathrm{Q})$ & FEFO & MILP & EX & & & & & $*$ & & $\mathrm{~N}$ \\
\hline $\begin{array}{l}\text { Hosseini-Motlagh et al. } \\
\text { (2020a) [18] }\end{array}$ & MP & SO & $\mathrm{OP}$ & FIFO & MILP & EX & & $\mathrm{S}, \mathrm{St}$ & & & & & Y \\
\hline $\begin{array}{l}\text { Hosseini-Motlagh et al. } \\
\text { (2020b) [32] }\end{array}$ & MP & MO & OP & FIFO & MILP & EX & & $\mathrm{S}, \mathrm{St}$ & $*$ & & & & Y \\
\hline $\begin{array}{l}\text { Attari \& Abdoli (2020) } \\
\text { [47] }\end{array}$ & MP & SO & $\mathrm{OP}$ & FIFO & MINLP & EX & & $S$ & $*$ & & & & $\mathrm{Y}$ \\
\hline $\begin{array}{l}\text { Derikvand et al. (2020) } \\
\text { [9] }\end{array}$ & MP & MO & $\mathrm{OP}$ & $\begin{array}{c}\text { Mixed } \\
\text { FIFO\& } \\
\text { LIFO }\end{array}$ & MILP & SIM & SL & $\mathrm{S}, \mathrm{St}$ & & $*$ & & & Y \\
\hline Current Research & MP & $\mathrm{MO}$ & $\mathrm{OP}$ & $\mathrm{H}$ & MILP & EX & SL,UL,SA,AP & $\mathrm{S}, \mathrm{St}$ & $*$ & $*$ & $*$ & $*$ & $\mathrm{Y}$ \\
\hline SP: Single Product & & \multicolumn{2}{|c|}{ H: Heuristic } & & \multicolumn{3}{|c|}{ SA: Substitution Allowance } & \multicolumn{3}{|c|}{ MDP: Markov Decision Process } & \multicolumn{3}{|c|}{ EOQ: Economic Order Quantity } \\
\hline MP: Multi Products & & \multicolumn{3}{|c|}{ MH: Metaheuristic } & \multicolumn{3}{|c|}{ AP: Assignment Policy } & \multicolumn{3}{|c|}{ FEFO: First-Expire-First-Out } & Y: Yes & & \\
\hline SO: Single Objective & & \multicolumn{3}{|c|}{ SIM: Simulation } & \multicolumn{3}{|c|}{ NLP: Nonlinear Programming } & \multicolumn{3}{|c|}{ FIFO: First-In-First-out } & $\mathrm{N}: \mathrm{No}$ & & \\
\hline MO: Multi Objectives & & \multicolumn{3}{|c|}{ SL: Shelf Life } & \multicolumn{3}{|c|}{ MILP: Mixed Integer Linear Programming } & \multicolumn{3}{|c|}{ LIFO: First-In-Last-Out } & St: Storage & & \\
\hline EX: Exact & & \multicolumn{3}{|c|}{ UL: Urgent Level } & \multicolumn{3}{|c|}{ DP: Dynamic Programming } & \multicolumn{3}{|c|}{ OP: Optimal Policy } & S: Supply & & \\
\hline
\end{tabular}


Table 2

\begin{tabular}{ccccccc}
\hline Objective & SO-E1 & SO-H1 & SO-H2 & SO-H3 & SO-H4 & MO \\
\hline E1 & 4325124 & 3933183 & 3766040 & 7877281 & 3044508 & 4413957 \\
H1 & 27966 & 27377 & 30123 & 0 & 30810 & 8730 \\
H2 & 281 & 209 & 0 & 480 & 120 & 114 \\
H3 & 342 & 0 & 20 & 256 & 0 & 22 \\
H4 & 0 & 12878 & 12941 & 18367 & 22306 & 6321 \\
MO & & & & & & 0.283 \\
\hline
\end{tabular}

Table 3

\begin{tabular}{cccccccccc}
\hline$(w 1, w 2, w 3, w 4, w 5)$ & $E 1$ & $H 1$ & $H 2$ & $H 3$ & $H 4$ & $Q_{p t}$ & $B_{p u t}$ & $X_{p u t} E_{p u t}$ \\
\hline$(1,0,0,0,0)$ & 3044508 & 30810 & 120 & 0 & 22306 & 9514 & 6162 & 0 & 0 \\
$(0.5,0.5,0,0,0)$ & 3882147 & 5340 & 215 & 3.69 & 28852 & 13301 & 1064 & 0 & 0 \\
$(0.4,0.3,0.1,0.1,0.1)$ & 4000081 & 8123 & 147 & 0 & 17642 & 13954 & 1625 & 0 & 0 \\
$(0.3,0.4,0.1,0.1,0.1$ & 4210884 & 5577 & 166 & 20 & 16151 & 1641 & 1116 & 0 & 0 \\
$(0.2,0.2,0.1,0.1,0.3)$ & 4633198 & 6752 & 198 & 3.16 & 4889 & 16031 & 1348 & 0 & 0 \\
$(0.2,0.2,0.2,0.2,0.2)$ & 4413957 & 8730 & 114 & 22 & 6321 & 15381 & 1746 & 0 & 0 \\
$(0.1,0.4,0.1,0.1,0.3)$ & 5022620 & 3153 & 196 & 0 & 3043 & 17154 & 631 & 0 & 0 \\
$(0,1,0,0,0)$ & 7877281 & 0 & 480 & 256 & 18367 & 13289 & 2911 & 2911 & 0 \\
$(0,0.5,0,0,0.5)$ & 8368413 & 0 & 579 & 178 & 0 & 15338 & 2978 & 2978 & 3 \\
$(0,0.4,0.1,0.1,0.4)$ & 8536463 & 38 & 2.37 & 1.69 & 28 & 14681 & 3617 & 3609 & 0 \\
$(0,0.3,0.3,0.1,0.3)$ & 7820352 & 107 & 1.66 & 2.59 & 77 & 15683 & 2615 & 2593 & 4 \\
$(0,0.25,0.25,0.25,0.25)$ & 8617606 & 107 & 1.66 & 1.19 & 77 & 13251 & 3760 & 3738 & 5 \\
$(0,0,1,0,0)$ & 3766040 & 30123 & 0 & 20 & 12941 & 10880 & 6025 & 0 & 0 \\
$(0,0,0,1,0)$ & 3933183 & 27377 & 209 & 0 & 13878 & 11336 & 5475 & 0 & 0 \\
$(0,0,0,0,1)$ & 3425124 & 27966 & 281 & 342.2 & 0 & 12717 & 5593 & 0 & 0 \\
\hline
\end{tabular}

Table 4

\begin{tabular}{ccccccccc}
\hline$(u 1, u 2, u 3)$ & $H 4$ & $H 3$ & $H 2$ & $H 1$ & $E 1$ & $B_{p u t}$ & $Q_{p t}$ & $Z_{p u t}$ \\
\hline$(10,10,10)$ & 4491788 & 18453 & 143 & 0 & 7859 & 15671 & 1848 & 0.82 \\
$(10,10,5)$ & 4398666 & 8633 & 134 & 5.735 & 7354 & 15189 & 1732 & 0.975 \\
$(10,7,7)$ & 4436957 & 12964 & 151 & 0 & 6704 & 15490 & 1856 & 0.777 \\
$(10,7,5)$ & 4413957 & 8730 & 114 & 22 & 6321 & 15381 & 1746 & 0.707 \\
$(10,5,5)$ & 4417825 & 8755 & 119 & 0.29 & 5593 & 15431 & 1754 & 0.713 \\
\hline
\end{tabular}

Table A.1

\begin{tabular}{ccccccccc}
\hline$\theta_{p p^{\prime} 3}$ & $p^{\prime}=1$ & $p^{\prime}=2$ & $p^{\prime}=3$ & $p^{\prime}=4$ & $p^{\prime}=5$ & $p^{\prime}=6$ & $p^{\prime}=7$ & $p^{\prime}=8$ \\
\hline$p=1$ & 1 & 0.9 & 0.8 & 0.8 & 0.9 & 0.8 & 0.8 & 0.3 \\
$p=2$ & 0 & 1 & 0 & 0.8 & 0 & 0 & 0 & 0.4 \\
$p=3$ & 0 & 0 & 1 & 0.9 & 0 & 0.8 & 0.8 & 0.5 \\
$p=4$ & 0 & 0 & 0 & 1 & 0 & 0 & 0 & 0.6 \\
$p=5$ & 0 & 0 & 0 & 0 & 0 & 0.9 & 0.9 & 0.7 \\
$p=6$ & 0 & 0 & 0 & 0 & 0 & 0 & 0 & 0.8 \\
$p=7$ & 0 & 0 & 0 & 0 & 0 & 0 & 0 & 0.9 \\
$p=8$ & 0 & 0 & 0 & 0 & 0 & 0 & 0 & 0 \\
\hline
\end{tabular}


Table A. 2

\begin{tabular}{ccccccccc} 
Cost & $p^{\prime}=1$ & $p^{\prime}=2$ & $p^{\prime}=3$ & $p^{\prime}=4$ & $p^{\prime}=5$ & $p^{\prime}=6$ & $p^{\prime}=7$ & $p^{\prime}=8$ \\
\hline$P C_{p t}$ & 320 & 180 & 205 & 190 & 280 & 188 & 204 & 210 \\
\hline
\end{tabular}

Table A.3

\begin{tabular}{|c|c|c|c|}
\hline Parameter & Value & Parameter & Value \\
\hline$T$ & $1,2, \ldots, 30$ & $F C$ & 50000 \\
\hline$R$ & $\subset T$, except $7^{\text {th }}$ day of each week & $P C$ & As Table A.3 \\
\hline$P$ & $\left\{\mathrm{O}^{-}, \mathrm{O}^{+}, \mathrm{A}^{-}, \mathrm{A}^{+}, \mathrm{B}^{-}, \mathrm{B}^{+}, \mathrm{AB}^{-}, \mathrm{AB}^{+}\right\}=\{1,2, \ldots, 8\}$ & $H C_{p i}$ & 25 \\
\hline$U$ & $\{1,2,3\}$ & $B C_{p t}$ & $3 * p c_{p t}$ \\
\hline$I$ & $5, \mathrm{i} \leq 3$ is considered as fresh & $E C_{p}$ & $p c_{p t}$ \\
\hline$v$ & 200000 & $E D_{p u t}$ & Uniform $(15,25)$ \\
\hline$m_{p}$ & 450 & $N D_{p u t}$ & Uniform $(1,10)$ \\
\hline$m v_{p}$ & 25000 & $C_{p t}$ & Uniform $(100,150)$ \\
\hline Demand Class & $\eta_{p u}$ & $\theta_{p p^{\prime} \mathrm{u}}$ & $\delta_{p u}$ \\
\hline$u 1$ & $0.6^{*} \theta_{p p^{\prime} 3}$ & 10 & 10 \\
\hline$u 2$ & $0.9 * \theta_{p p^{\prime} 3}$ & 7 & 7 \\
\hline$u 3$ & As Table A. 2 & 5 & 5 \\
\hline
\end{tabular}

Table A.4

\begin{tabular}{ccccccccc}
\hline$S^{p p^{\prime}}$ & $p^{\prime}=1$ & $p^{\prime}=2$ & $p^{\prime}=3$ & $p^{\prime}=4$ & $p^{\prime}=5$ & $p^{\prime}=6$ & $p^{\prime}=7$ & $p^{\prime}=8$ \\
\hline$p=1$ & 1 & 1 & 1 & 1 & 1 & 1 & 1 & 1 \\
$p=2$ & 0 & 1 & 0 & 1 & 0 & 0 & 0 & 1 \\
$p=3$ & 0 & 0 & 1 & 1 & 0 & 0 & 1 & 1 \\
$p=4$ & 0 & 0 & 0 & 1 & 0 & 0 & 0 & 1 \\
$p=5$ & 0 & 0 & 0 & 0 & 0 & 1 & 1 & 1 \\
$p=6$ & 0 & 0 & 0 & 0 & 0 & 0 & 0 & 1 \\
$p=7$ & 0 & 0 & 0 & 0 & 0 & 0 & 0 & 1 \\
$p=8$ & 0 & 0 & 0 & 0 & 0 & 0 & 0 & 1 \\
\hline
\end{tabular}

